Review

\title{
Photosensitization and controlled photosensitization with BODIPY dyes
}

\author{
Abdurrahman Turksoy ${ }^{\mathrm{a}}$, Deniz Yildiz ${ }^{\mathrm{a}}$, Engin U. Akkaya ${ }^{\mathrm{a}, \mathrm{b}, *}$ \\ a Department of Chemistry, Bilkent University, 06800 Ankara, Turkey \\ ${ }^{\mathrm{b}}$ UNAM-National Nanotechnology Research Center, Bilkent University, 06800 Ankara, Turkey
}

\section{A R T I C L E I N F O}

\section{Article history:}

Received 18 July 2017

Received in revised form 27 September

2017

Accepted 29 September 2017

Available online 26 October 2017

\section{Keywords:}

Photosensitization

BODIPY

Photodynamic therapy

Reactive oxygen species

Selective photosensitizers

\begin{abstract}
A B S T R A C T
Highly versatile BODIPY dyes proved themselves to be very useful as photosensitizers. These dyes can be derivatized to absorb essentially anywhere in the visible the near IR region of the spectrum. As a result of their diverse reactivity, singlet oxygen generation efficiency can be modulated very precisely, leading to a number of selective photosensitizers for photodynamic therapy. Among the biologically relevant modulators, glutathione concentration and $\mathrm{pH}$ received particular attention. In this review, we highlight modulatable BODIPY-based photodynamic photosensitizers, and various synthetically useful chemical reactions triggered by singlet oxygen and other reactive oxygen species generated by BODIPY-based photosensitizers.
\end{abstract}

(ㄷ) 2017 Elsevier B.V. All rights reserved.

\section{Contents}

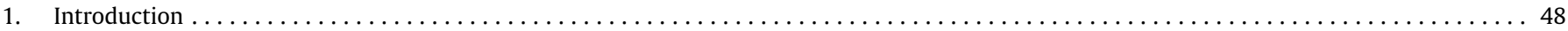

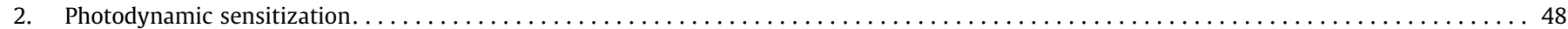

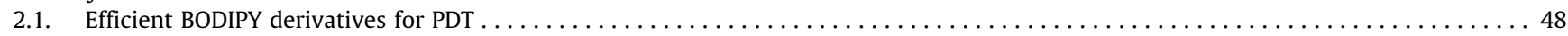

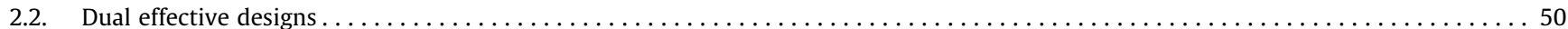

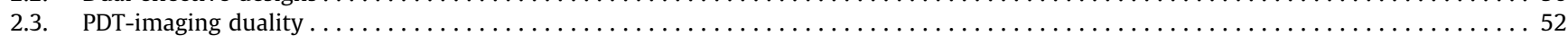

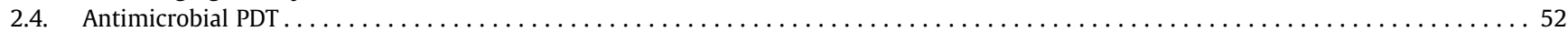

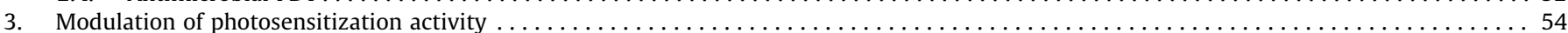

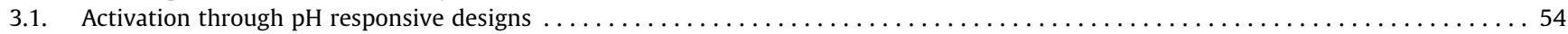

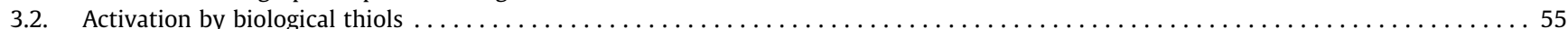

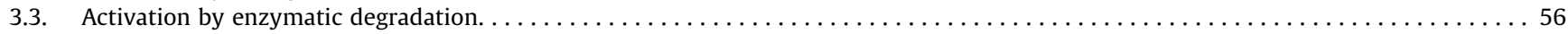

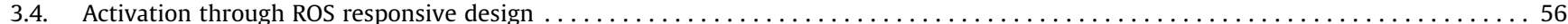

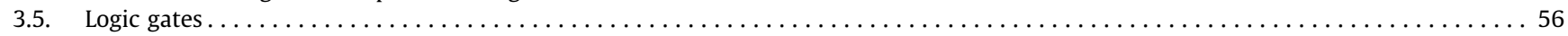

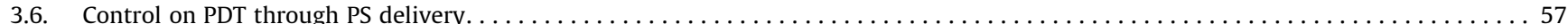

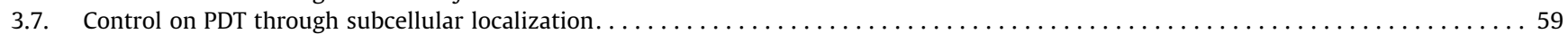

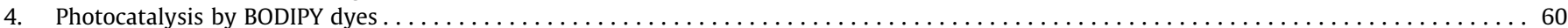

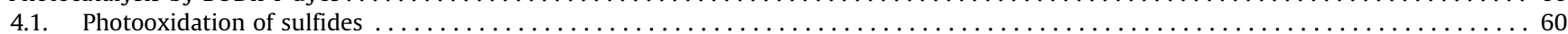

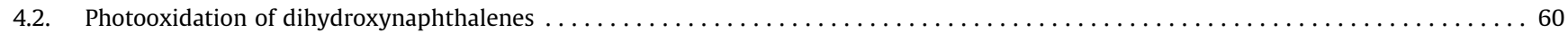

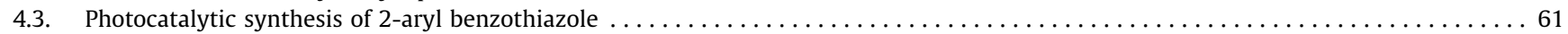

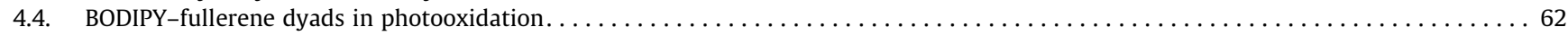

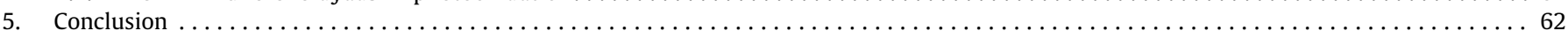

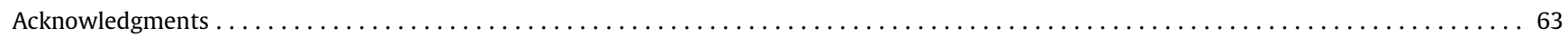

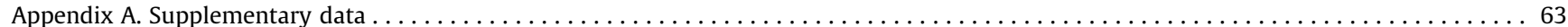

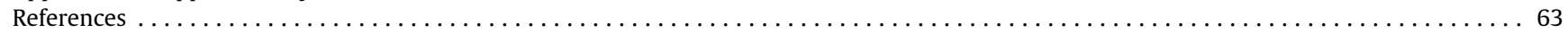

\footnotetext{
* Corresponding author at: Department of Chemistry, Bilkent University, 06800 Ankara, Turkey.

E-mail address: eua@fen.bilkent.edu.tr (E.U. Akkaya).
} 


\section{Introduction}

Although structurally related to porphyrins, the parent BODIPY compound is not particularly efficient as a photosensitizer. Typically these dyes are known to be highly fluorescent, and the intersystem crossing to the triplet manifold is ineffective. However, intersystem crossing rate can be enhanced by heavy atom substitution, or by the special arrangement of the BODIPY chromophores. Bromine or iodine substitution at the BODIPY core is very straightforward to enhance singlet oxygen generation. The interplay of various excited state processes such as fluorescence, intersystem crossing, through-bond and through space energy transfers, photoinduced electron transfer and non-radiative relaxations make modulatable photosensitization possible. Reversible or irreversible changes in the structures of photosensitizers as a result of interaction with chemical and/or biological modulators open a path for such control in activity. Thus, a spatial and temporal control of photosensitizer action becomes possible, which is an important issue in the photodynamic therapy of cancers.

Reactive oxygen species (ROS), and specifically singlet oxygen is also important in synthetic transformations. BODIPY based photosensitizers proved useful in this way as well, because of the fact that the excitation wavelength and other properties can be adjusted in a very straightforward manner.

\section{Photodynamic sensitization}

Starting from the beginning of 20th century photodynamic therapy (PDT) has been recognized as an alternative treatment modality for various types of cancer [1-4]. Even though the first experiments to cure cancer date back to 1903, modern treatment of cancer cells with photodynamic therapy started in mid-20th century with the synthesis and application of compound called 'haematoporphyrin' known as HPD [3]. In 1975, Thomas Dougherty et al. reported the elimination of mammary tumor growth with HPD and red light in mice [5]. Following this initial demonstration of PDT in mice, patients of bladder cancer became first subjects of human trials in 1976 [6]. Afterwards, lung tumors [7], esophageal cancer [8] and gastric carcinoma [9] patients were treated with PDT and promising responses were obtained from early-stage patients [3]. In subsequent studies PDT was utilized as a treatment method for following tumors and cancer types: brain tumors [10-12], intraocular cancer [13-15], breast cancer [16-18], head and neck tumors [19,20], pancreatic cancer [21], gynecological tumors [22,23].

Photodynamic therapy requires basically three elements: photosensitizer, light and oxygen. Effective treatment requires the combination of each element at the cellular level. The prerequisite of PDT imposed several properties on photosensitizers, evolving into the concept of ideal photosensitizer characterized by followings: low dark toxicity, red or Near-IR absorption, high absorption coefficient ( $>20,000 \mathrm{M}^{-1} \mathrm{~cm}^{-1}$ ), ease of synthesis, known composition, long shelf-life, easy elimination from patient, and high singlet oxygen quantum yield. Therefore, photodynamic therapy modality relies heavily on the properties of administered photosensitizer. The first approved photosensitizer in clinical use was Photofrin ${ }^{\circledR}$ which has several disadvantages ranging from shorter wavelength absorption compared to optical window to prolonged skin sensitivity [24]. This has motivated researchers to develop advanced photosensitizers having superior photophysical properties. BODIPY dyes stand out in means of preferable photophysical properties, yet without imperative functionalization BODIPY dyes are not appropriate for PDT application due to their highly fluorescent nature. It was envisioned that analysis of BODIPY derivatives with distinct functionalization methods is imperative, yet discussion is constrained to only phototherapeutic cases.

\subsection{Efficient BODIPY derivatives for PDT}

The highly fluorescent BODIPY (4,4-difluoro-4-borata-3a-azo nia-4a-aza-s-indacene) unit which has similar structure with porphyrin was designed and synthesized by Treibs and Kreuzer in 1968 [25]. The parent BODIPY unit, having a major absorption peak corresponding to $S_{0}-S_{1}$ transition near $500 \mathrm{~nm}$, can be functionalized with heavy halogen atoms such as bromine and iodine so as to enhance intersystem crossing efficiency, increasing singlet oxygen quantum yield, through increase in spin-orbit coupling as reported by O'Shea in aza-BODIPY and Nagano in BODIPY derivatives $[26,27]$. However, incorporation of halogen and other heavy atoms to enhance intersystem crossing efficiency to produce singlet oxygen leads to increase in dark toxicity. That is, phototherapeutic agent induces damage to normal cells as well. Akkaya and co-workers reported orthogonal BODIPY structure $\mathbf{1}$ which generates singlet oxygen in the absence of heavy atoms due to peculiar nature of first excited state with tetraradicalic character as discussed in detailed manner in follow-up theoretical studies $[28,29]$. Further cell culture experiments of micelle-embedded dyes with K562 human erythroleukemia validated likely usage in phototherapeutic applications with $\mathrm{EC}_{50}$ (median effective concentration) value of $50 \mathrm{nM}$ after PDT treatment without observable dark toxicity. Zhang and Yang reported the dimer, which is no longer necessarily being orthogonal, produced singlet oxygen only in nonpolar solvents due to fast internal charge transfer which renders intersystem crossing noncompetitive, indicating possible control mechanism on phototherapeutic treatment modality [30]. However, the major drawback of orthogonal BODIPYs in PDT application is being incompetent singlet oxygen generator under longer wavelength of light exposure. Moreover, extension of $\pi$-conjugation on one of the BODIPY dyes was found to disrupt symmetry, leading formation of non-degenerate HOMOs and LUMOs which eventually gives rise to poor ISC [31]. Akkaya and co-workers also reported merging orthogonal BODIPYs with upconverting nanoparticles (UCNPs) enabled generation of singlet oxygen at comparably longer wavelength of light; $980 \mathrm{~nm}$ [32]. Working principle relied on the emission from UCNPs at shorter wavelengths upon exposure to NIR light through multitude of metastable states with relatively long lifetimes. Covalent functionalization of UCNPs yielding energy transfer from UCNPs to orthogonal BODIPYs resulted in the excitation of BODIPY dyes with NIR light source, producing singlet oxygen with biocompatible BODIPY functionalized nanoparticles. Another approach toward formation of singlet oxygen without utilizing heavy atoms is dimeric structure of BODIPY dyes reported by Krüger and co-workers [33]. Dimeric structure $\mathbf{2}$ was reported to have comparable singlet oxygen quantum yields in toluene and dichloromethane; however, negligible singlet oxygen quantum yield was observed in acetonitrile, exhibiting solvent dependence of photophysical properties of 2. Besides, orthogonal BODIPY trimer $\mathbf{3}$ was shown to generate singlet oxygen as well with working principle similar to orthogonal BODIPY dimers [34] (Fig. 1).

Photosensitizers need to be absorbing in therapeutic window for clinical application purposes. There are several methods to modify BODIPY dyes, one of which is fusion of aromatic groups into the structure through several mechanisms. Recently Hao and co-workers reported facile, region-selective and stepwise synthesis of several BODIPY derivatives through $\mathrm{C}-\mathrm{H}$ arylation with Suzuki, Stille, Heck and Sonogashira coupling reactions [35]. All $\alpha$-arylated structures showed red-shifted absorption/emission, but 2-methylfuran 4, 4-ethynylanisole 5 and 2-ethynylthiophene 6 were reported have absorption maxima around $670 \mathrm{~nm}$ due to their electron rich and higher $\pi$-conjugation capabilities compared to thiophene and phenyl derivatives placed at 3 and 5 positions of the dye. Singlet oxygen quantum yield experiments with 
1,3-diphenylisobenzofuran (DPBF) indicated comparable singlet oxygen generation ability of these dyes (Fig. 2).

Also, Jiao and Hao reported pyrrole fused at 3 and 5 positions of BODIPY dyes with several modifications in terms of types of pyrrole and substituted groups on BODIPY [36]. Addition of each pyrrole unit shifted absorption/emission maxima towards NIR region such that structure 7 has absorption maxima at $745 \mathrm{~nm}$. It was also reported that addition of bromine atoms into the structure not only pronounces heavy atom effect but also shifts absorption maxima towards NIR region as can be realized in the comparison of 7 and 8. Moreover, alkyl substitutions on pyrrole moieties were reported to decrease singlet oxygen generation efficiency and fluorescence. However, singlet oxygen generation efficiency of the dyes $\mathbf{9}$ and $\mathbf{1 0}$ were found to be fairly comparable with methylene blue along with good fluorescence quantum yields, indicating their potential usage in PDT and imaging purposes (Fig. 3).
Another versatile functionalization of BODIPY derivatives was reported by You and co-workers [37]. In this particular study thieno-pyrrole-fused BODIPY $\mathbf{1 1}$ was utilized as a platform to obtain NIR region absorbing/emitting dyes. Participation of $\mathbf{1 1}$ in Suzuki, Stille and Heck coupling reaction enabled wide range of functionalization desired for NIR dyes. Functional group tolerating nature of Suzuki coupling reactions offered preparation of highly conjugated systems in wide scope of chemical scaffolds. Besides, Heck coupling enabled to reach extended $\pi$-conjugation for further red-shift. Resultant structures from such chemistry displayed absorption maxima ranging from $650 \mathrm{~nm}$ to $850 \mathrm{~nm}$ which is rare case in BODIPY dye derivatives. Along with such spectral shift, derivatives of thieno-pyrrole-fused BODIPY 11, exhibiting comparable singlet oxygen generation and fluorescence emission that can also be utilized in imaging purposes, provide promising future. You and co-workers also reported that similar BODIPY derivatives

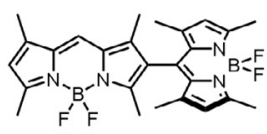

$$
\begin{gathered}
\boldsymbol{K}_{\text {abs }}=\mathbf{5 1 4} \mathrm{nm} \\
\boldsymbol{K}_{\text {ems }}=527 \mathrm{~nm} \\
\Phi \Delta=0.51, \mathrm{CH}_{2} \mathrm{Cl}_{2}
\end{gathered}
$$

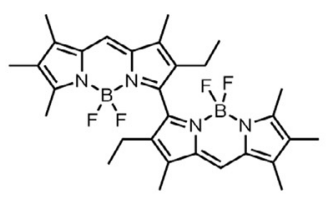

2

$$
\begin{aligned}
\kappa_{\mathrm{abs}} & =565 \mathrm{~nm} \\
\boldsymbol{K}_{\mathrm{ems}} & =648 \mathrm{~nm}
\end{aligned}
$$$$
\Phi \Delta=0.50, \mathrm{CH}_{2} \mathrm{Cl}_{2}
$$

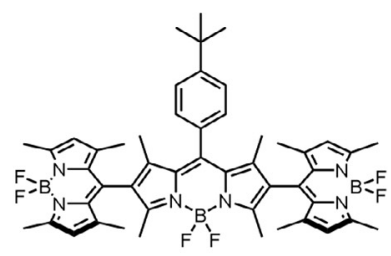

3

$\begin{aligned} \boldsymbol{K}_{\mathrm{abs}} & =\mathbf{5 0 7} \mathbf{~ n m} \\ \boldsymbol{K}_{\mathrm{max}} & =\mathrm{ND}\end{aligned}$ $\boldsymbol{K}_{\text {ems }}=\mathrm{ND}$
$\Delta=0.53, \mathrm{CH}_{2} \mathrm{C}$

Fig. 1. Heavy atom free singlet oxygen producing BODIPY derivatives 1-3 (orthogonal BODIPY derivatives).

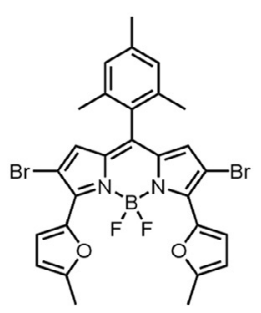

4

$\kappa_{\text {abs }}=667 \mathrm{~nm}$

$\hat{K}_{\text {ems }}=689 \mathrm{~nm}$ $\Phi \Lambda=0.14, \mathrm{CH}_{2} \mathrm{Cl}_{2}$

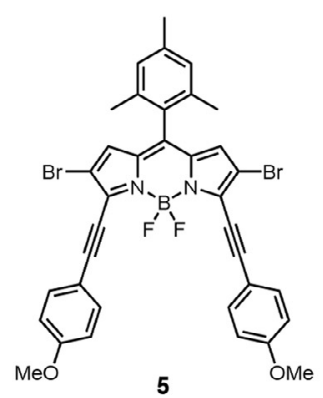

$\Lambda_{\text {abs }}=667 \mathrm{~nm}$

$\hat{\Lambda}_{\text {ems }}=697 \mathrm{~nm}$ $\Phi \mathrm{f}=0.48, \mathrm{CH}_{2} \mathrm{Cl}_{2}$

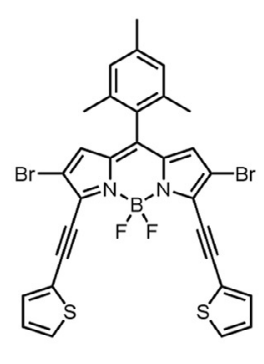

6

$\Lambda_{\text {abs }}=667 \mathrm{~nm}$

$\hat{K}_{\text {ems }}=706 \mathrm{~nm}$

$\Phi \Lambda=0.19, \mathrm{CH}_{2} \mathrm{Cl}_{2}$

Fig. 2. Longer wavelength absorbing/emitting, aromatic group fused (through various coupling methods) BODIPY derivatives 4-6.<smiles></smiles>

7

$$
\begin{gathered}
\hat{\Lambda}_{\text {abs }}=745 \mathrm{~nm} \\
\hat{\Lambda}_{\text {ems }}=818 \mathrm{~nm} \\
\Phi \mathrm{f}=<0.001, \mathrm{CHCl}_{3}
\end{gathered}
$$

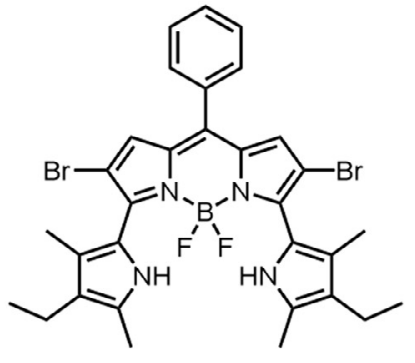

8

$$
\begin{aligned}
& \Lambda_{\mathrm{abs}}=729 \mathrm{~nm} \\
& \boldsymbol{\Lambda}_{\mathrm{ems}}=807 \mathrm{~nm}
\end{aligned}
$$$$
\Phi f=0.02, \mathrm{CHCl}_{3}
$$<smiles></smiles>

9

$$
\begin{aligned}
\hat{\Lambda}_{\text {abs }} & =682 \mathrm{~nm} \\
\hat{\Lambda}_{\text {ems }} & =717 \mathrm{~nm}
\end{aligned}
$$$$
\Phi \Delta=0.23, \mathrm{C}_{6} \mathrm{H}_{6}
$$<smiles></smiles>

10

$\hat{\Lambda}_{\mathrm{abs}}=685 \mathrm{~nm}$

$\hat{\Lambda}_{\text {ems }}=720 \mathrm{~nm}$

$\Phi \Delta=0.39, \mathrm{C}_{6} \mathrm{H}_{6}$

Fig. 3. Longer wavelength absorbing/emitting, pyrrole fused BODIPY derivatives 7-10. 
12 and 13 produced singlet oxygen upon exposure to light above $700 \mathrm{~nm}$ along with being relatively high resistant to photobleaching, demonstrating desirable properties for use in PDT [38]. Moreover, Shen and co-workers reported unsymmetrical thieno-pyrrole-fused BODIPY derivative 14, absorbing above $700 \mathrm{~nm}$, as a powerful example of PDT agent supported with HeLa cell culture experiments, resulting in $\mathrm{IC}_{50}$ (half maximal inhibitory concentration) value of $7.12 \mu \mathrm{M}$ without observable dark toxicity [39] (Fig. 4).

Direct functionalization of BODIPY dyes, obtained from 2,4dimethyl pyrrole, with styryl moieties due to acidic nature of hydrogen atoms on 3 and 5 positions was achieved via Knoevenagel condensation [40,41]. Further efforts enabled to obtain tetra-styryl BODIPY derivatives [42]. Addition of each styryl unit shifted $S_{0}-S_{1}$ absorption band of BODIPY toward NIR region which is imperative for PDT applications. To illustrate possible usage of such derivatives in vitro Akkaya and co-workers reported water soluble di-styryl BODIPY 15 decreased viability of K562 human erythroleukemia cells with $\mathrm{EC}_{50}$ value of less than $200 \mathrm{nM}$ without observable dark toxicity [43]. Moreover, in order to obtain NIR absorption/emission nitrogen atom can be incorporated on meso position of BODIPY dyes, forming so called aza-BODIPY dye. In fact, changing meso atom from carbon to nitrogen tends to narrow the HOMO-LUMO band gap, enabling longer wavelength absorption. O'Shea and co-workers reported in vitro photo-therapeutic effects of aza-BODIPY 16 by exhibiting cell viability decrease of HeLa cervical carcinoma and MRC5-SV40 transformed fibroblast cancer cell lines with $\mathrm{EC}_{50}$ values of $41 \pm 3 \mathrm{nM}$ and $14 \pm 1 \mathrm{nM}$ at higher light dosages, respectively [26]. Additionally, it has been reported that incorporation of aryl substituents on aza-BODIPY dyes shifted absorption maxima above $700 \mathrm{~nm}$ for practical applications in PDT [44] (Fig. 5).

\subsection{Dual effective designs}

Photodynamic therapy is a well-established treatment modality depending on formation of reactive oxygen species through
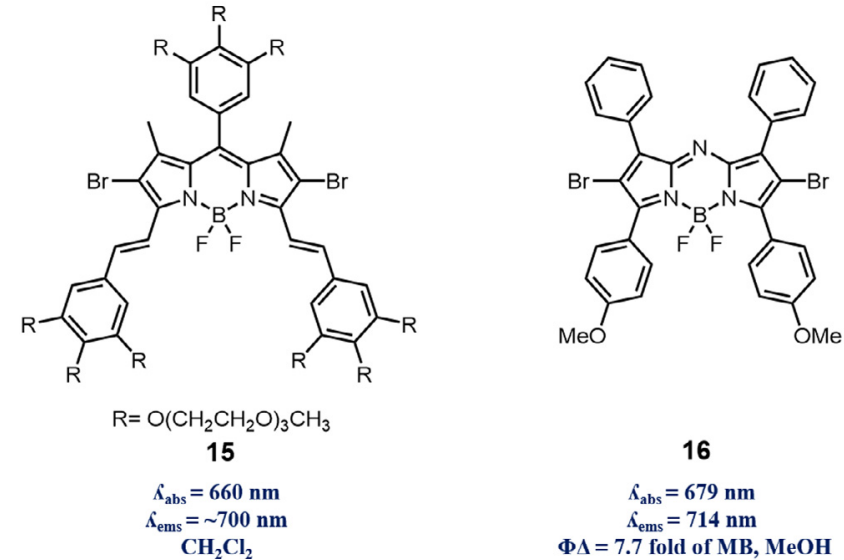

Fig. 5. BODIPY 15 functionalized with Knoevenagel condensation. Longer wavelength absorbing/emitting, heavy atom incorporated aza-BODIPY 16.

competitive Type I and II mechanisms. It has been reported that vast majority of photosensitizers follow Type II mechanism, forming singlet oxygen as main cytotoxic agent [45]. BODIPY type dyes have been considered to perform with same mechanism until the Wang and co-workers reported first observation of DNA cleavage through formation of reactive oxygen species other than singlet oxygen with BODIPY type dyes [46]. Prepared di-styryl and dihalogenated BODIPY dyes 17 and 18, proven to bind CT DNA with absorption titration, circular dichroism and viscosity experiments, successfully cleaved CT DNA. In contrast to 18, $\mathrm{NaN}_{3}$ and KI restricted photo-activity of $\mathbf{1 7}$, indicating exertion of photoactivity through both Type I and Type II mechanisms. Even though these mechanisms are competitive in nature, Wang et al. pointed that BODIPY dyes can perform as both types. Such duality stems from intrinsic property of photosensitizers; however, rational designs can enhance PDT with release of other reactive species in a synergetic manner. Sortino and co-workers designed BODIPY derivative<smiles></smiles>

11 $\hat{\Lambda}_{\text {abs }}=635 \mathrm{~nm}$
$\Lambda_{\text {ems }}=650 \mathrm{~nm}$
$\Phi \Delta=0.52, \mathrm{CHCl}_{3}$<smiles>COc1ccc(-c2sc3c(c2Br)=c2c(c(C(F)(F)F)c4cc5sc(-c6ccc(OC)cc6)c(Br)c5n24)C=3)cc1</smiles>

12

$\kappa_{\text {abs }}=720 \mathrm{~nm}$

$\boldsymbol{\Lambda}_{\text {ems }}=754 \mathrm{~nm}$

$\Phi \Delta=1.2$ fold of CMP, THF<smiles></smiles>

14

$\hat{K}_{\mathrm{abs}}=698 \mathrm{~nm}$

$\hat{\Lambda}_{\mathrm{ems}}=724 \mathrm{~nm}$

$\Phi \Delta=0.63, \mathrm{CH}_{2} \mathrm{Cl}_{2}$<smiles></smiles>

13

Fig. 4. Longer wavelength absorbing/emitting, thieno-pyrrole fused BODIPY derivatives 11-14. 
19 with NO photodonor (NOPD); trifluoromethyl substituted nitroaniline [47]. Mechanism of NOPD relies on photorearrangement prior to NO release with resultant formation of phenol derivatives. Excitation of structure with blue and green light sources produced NO and singlet oxygen, respectively. Cell viability experiments conducted with melanoma carcinoma cells proved the dual nature of this design such that depending on irradiation wavelength formed reactive species decreased cell viability greatly. On the other hand, in order to increase the efficiency of PDT instead of using combinatory effects of reactive species, intrinsic limitations of PDT can be overcome. It has been reported that PDT diminishes oxygen level inside the cell by simply consuming dissolved oxygen to generate singlet oxygen, and also destroying vasculature of tumor tissue feeding cells, making cell environment even more hypoxic [48-50]. Such induction of more hypoxic environment renders PDT self-limiting. Fractional photodynamic therapy, which delivers light fractionally to allow cells replenish oxygen levels during dark cycle of treatment, is promising so as to eliminate such intrinsic limitation. Akkaya and co-workers reported that singlet oxygen storage unit (2-pyridone) containing, longer wavelength absorbing/emitting BODIPY dye derivative 20 produces singlet oxygen under light irradiation (light cycle of FPDT) and stores some of it in the storage unit [51]. During dark cycle of FPDT, which is not utilized for treatment purposes, with thermal cycloreversion formed endoperoxide structure released singlet oxygen while cells replenish their oxygen level to keep treatment ongoing. Such dual effective nature of $\mathbf{2 0}$, being validated with HeLa cancer cell culture experiments indicating $\mathrm{CC}_{50}$ ( $50 \%$ cytotoxic concentration) value of $8.6 \mathrm{nM}$, represents promising future as s FPDT drug (Fig. 6).

Photodynamic therapy requires penetration of light through turbid environment in tumor tissue. Several reports have agreed upon best penetration of light through tissue is observed wavelengths between approximately $700-1000 \mathrm{~nm}$; therapeutic window [52,53]. Considering light penetration issue and intrinsic hypoxia-induction by PDT as mentioned before, Chen and coworkers reported dual active agent that can go through PDT along with PTT [54]. That is, BODIPY embedded into polymeric vesicles can absorb light at/under $660 \mathrm{~nm}$. Even though, such design can produce singlet oxygen efficiently upon exposure to light at $660 \mathrm{~nm}$, vesicle structure can absorb light at $785 \mathrm{~nm}$ which is much more penetrable through tissue and produces lower dosages of PDT along with hypothermia through non-radiative relaxation of BODIPY dyes from first excited singlet state to ground state. PDTsynergized PTT treatment induced total tumor ablation along with several characteristic features to address some forenamed issues;

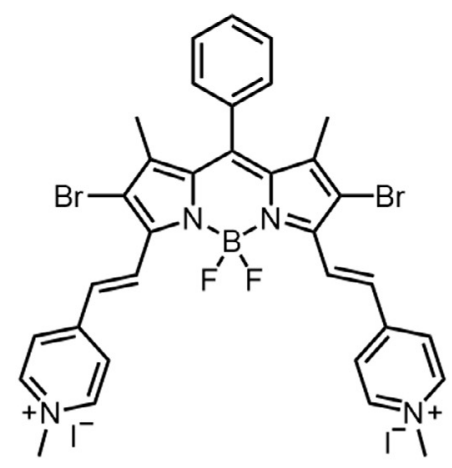

17

$$
\begin{gathered}
\hat{\Lambda}_{\mathrm{abs}}=648 \mathrm{~nm} \\
\hat{\Lambda}_{\text {ems }}=680 \mathrm{~nm} \\
\Phi \Delta=0.10, \text { PBS Buffer }
\end{gathered}
$$

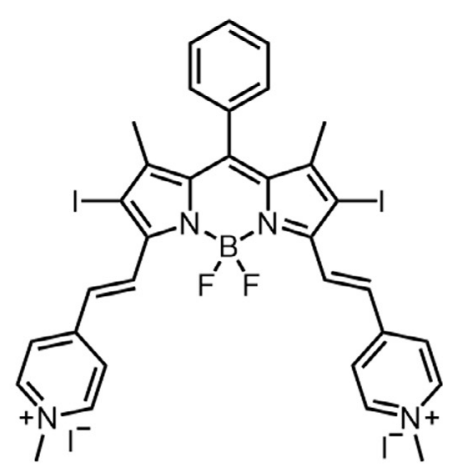

18

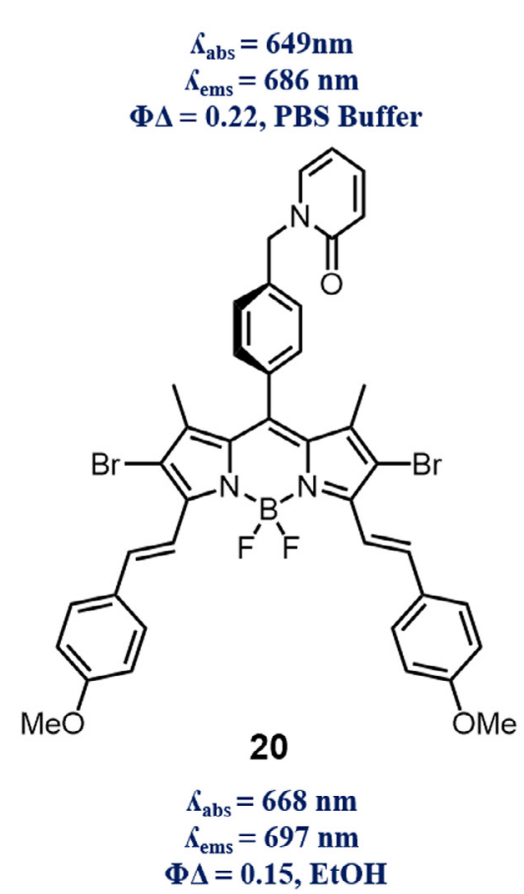

Fig. 6. BODIPY derivatives 17-20 for dual effective designs. 
preferable cellular uptake, enhance tumor accumulation, economy in usage of cellular oxygen and deeper light penetration. Another synergetic demonstration of PDT-PTT dual treatment method based on aza-BODIPY reported by Dong and co-workers [55]. Phototherapeutic performance of self-assembled cubic nanoparticles from IABDP with DSPE-mPEG 2000 were exploited by using Xenon lamp (indicating no specific light source is required such as laser) as the light source in vivo and in vitro studies, leading to the conclusion of this dual therapeutic treatment modality being remarkably phototoxic such that tumor growth potently can be suppressed without any apparent side effect. In addition, photodynamic therapy can be employed with other treatment modalities like chemotherapy to synergistically destroy tumor tissue. Hu and Wang reported very recent demonstration of such duality. Vesicles formed from BODIPY dye and pillar[5]arene with host-guest chemistry can be efficaciously loaded with chemotherapy drug DOX [56]. The cytotoxicity analysis of vesicles with A549 lung adenocarcinoma cell line pointed out that, due to intrinsic low-pH nature, cancer cell leads release of DOX with destruction of vesicle along with phototherapeutic effect of photosensitizer, causing reduction in cell viability with $\mathrm{IC}_{50}$ values of $1.40 \mu \mathrm{M}$ under light irradiation and $5.50 \mu \mathrm{M}$ under dark. Another PDT/chemotherapy treatment duality was reported by Yan and co-workers depending on release of DOX and phototherapeutic agent in cancer cells due to acidic nature of microenvironment of tumor tissue and cancer cells. DOX polymeric micelles entrapped BODIPY dyes were prepared and evaluated with HepG2 cell lines [57]. Cell culture experiments validated treatment duality based on release of chemotherapy drug and PDT drug inside cancer cells by indicating immense difference in cell viability under light and dark conditions.

\subsection{PDT-imaging duality}

In previous section, synergetic treatment designs were discussed for enhancement of PDT, yet it is of utmost importance to be able to measure the effect of phototherapeutic modalities directly rather than measuring biological effect on a cellular level which is the cumulative result; cell death. Therefore, structural construction can be accomplished by combining photosensitizer and imaging units since triplet state accumulation required for PDT will eventually quench fluorescence of chromophore, reducing its imaging competence. Erbas-Cakmak and Akkaya reported proof of principle PDT/imaging design 21 consisting of a photosensitizer and the fluorophore [58]. Covalently combined BODIPY units performed electronic energy transfer from fluorophore (donor) to photosensitizer (acceptor) such that upon excitation of fluorophore at shorter wavelength of light yielded characteristic fluorescence emission of photosensitizer. However, exposure to longer wavelength of light, that can excite photosensitizer and generate singlet oxygen, resulted recovery of fluorescence of the donor unit, indicating the working principle. That is, upon fracture of singlet oxygen labile linker enhancement of fluorescence of donor indicated formation of main cytotoxic agent in a self-reporting manner.

Employment of biological thiols (GSH or Cys) in activation of several designs can also be exploited in PDT-imaging modality. $\mathrm{Wu}$ and co-workers reported the BODIPY dyad $\mathbf{2 2}$ was designed to contain photosensitizer and fluorophore as being donor and acceptor, respectively [59]. Therefore, singlet oxygen generation capability of photosensitizer was quenched since accumulation on triplet state is not allowed through FRET mechanism. On the other hand, fluorescence of acceptor unit was caged with DNBS (2,4-dinitrobenzenesulfonyl), leading that PDT and imaging capability are not pronounced; however, dyad contains disulfide linker which can be cleaved by biologicals thiols. Wu et al. reported that in HeLa cells existent biological thiol derivatives uncaged PDT and imaging units of $\mathbf{2 2}$ through cleavage of di-sulfide bond (inactivates FRET) and removal of DNBS (restores fluorescence) such that singlet oxygen and fluorescence quantum yield increased from $16.7 \%$ and $1.3 \%$ to $71.5 \%$ and $47.6 \%$, respectively. Based on these reports, it is worth mentioning that combination of a reporter and a photosensitizer much more auspicious method, yet usage of single chromophore cannot be underestimated. However, for PDT/imaging duality, it is compulsory to balance the capability of singlet oxygen generation and fluorescence of photosensitizers along with high extinction coefficients at relatively long wavelength compared to optical window. One of recent examples of single chromophore containing PDT/imaging duality was reported by You and co-workers. Halogen atom free BODIPY dye $\mathbf{2 3}$ having singlet oxygen quantum yield of 0.42 and moderate fluorescence quantum yield 0.22 was proven to be phototoxic in a cell culture experiments with colon-26 cells ( $\mathrm{IC}_{50}$ value of $3.0 \mu \mathrm{M}$ after irradiation without any dark toxicity up to $5.0 \mu \mathrm{M}$ ) and an antitumor agent in demonstration with Balb/c tumor-bearing mice along with live mice optical imaging, indicating achievement of PDT/imaging duality with single chromophore [60] (Fig. 7).

\subsection{Antimicrobial PDT}

Novel treatment strategies are highly demanding for the infectious diseases despite the focused continuous research on antibiotics and vaccines, such as modifying the structures of traditional antibiotics, because of the fact that the prokaryotic organisms can easily develop resistance against the new agents. Antimicrobial-PDT, which is also called as PACT (photodynamic antimicrobial chemotherapy) is a promising alternative to traditional chemotherapies. Since photodynamic action is based on oxidative damaging in bacterial membrane resulting the cytotoxic effects, it can be used for all types of microorganisms regardless of resistance obstacle. There are several examples of APDT with chlorin ${ }_{\mathrm{e} 6}$ or porphyrin derivatives [61-63]; however, BODIPY dyes are indeed promising due to low dark-toxicity and ease of functionalization. Some cationic BODIPY derivatives are developed for that purpose as shown in Fig. 8.

The idea behind the cationic structure is that positive charge on the photosensitizer can promote stronger electrostatic interaction with negatively charged bacterial cell wall. To ensure selectivity towards pathogens, human MDA-MB-231 cell lines are also incubated with compound $\mathbf{2 4}$, by O'Shea and co-workers and results showed only minimal binding to human cell lines compared to Gram-positive Staphylococcus aureus, Gram-negative Escherichia coli and Candida albicans yeast cells [64]. Additionally, compound 24 showed no dark toxicity in the absence of light with $1 \mu \mathrm{g} / \mathrm{mL}$ dosage; however, with introduction of light, reduction in the viability was observed $\left(2,4,8,16 \mathrm{~J} / \mathrm{cm}^{2}\right.$ irradiation causes $\log _{10}$ reduction of $0.1,0.3,1.4,3.0$, respectively). Among others, compound 25 showed the lowest photodynamic minimal bactericidal concentration value, $1.66 \mu \mathrm{M}$, for the $E$. coli. (whereas intrinsic toxicity was observed up to $40 \mu \mathrm{M}$.) Banfi and his co-workers compared the efficiency of compound $\mathbf{2 5}$ with compound $\mathbf{2 6}$, only methyl pyridinium cationic moiety is changed with benzyl pyridinium, concluding that masking the positive charge by benzylic unit prevents the photosensitizer from coordinating tightly on the bacterial cell wall resulting around 4-fold less efficiency [65]. Due to the excellent APDT efficiency of compound $\mathbf{2 5}$ on E. coli and Staphylococcus xylosus, Ghiladi and his co-workers conducted the experiments on eight bacteria strains including Gram-positive, Gram-negative and drug-resistant bacteria, three yeast species and three viruses with promising results so that compound $\mathbf{2 5}$ is able to mediate the photodynamic inactivation at nanomolar concentrations with short light dosages, meaning it is more efficient compared to methylene blue and TMPyP [66]. Barbieri and Orlandi tested the photodynamic 


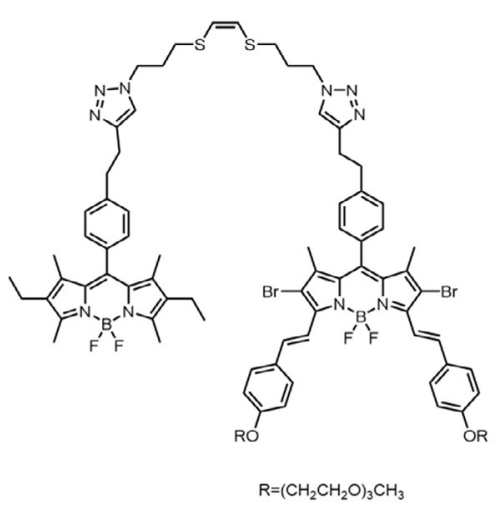

21

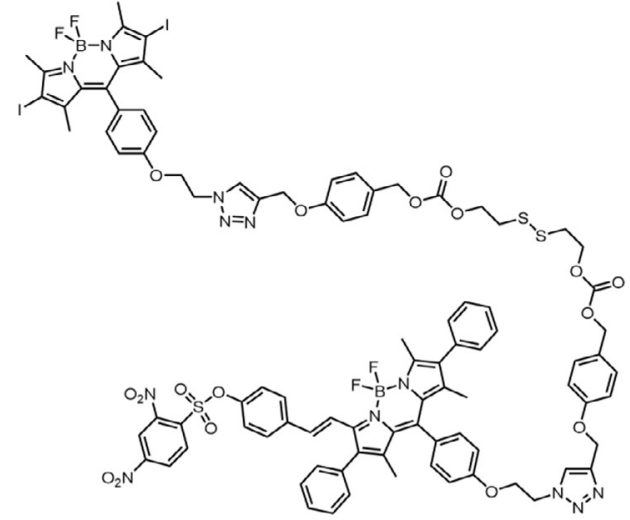

22

$\kappa_{\mathrm{abs}}=535,580 \mathrm{~nm}$

$\begin{aligned} \hat{A}_{\text {ens }} & =554,622 \mathrm{~nm} \\ \Phi \Delta=0.17, \mathrm{DMF} / \mathrm{War} & \end{aligned}$

(in the absence of Cys)

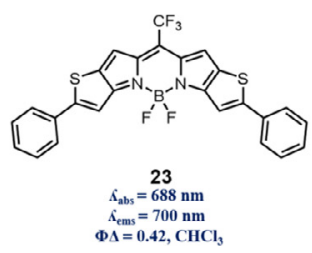

Fig. 7. BODIPY designs 21-23 for PDT/imaging modality.

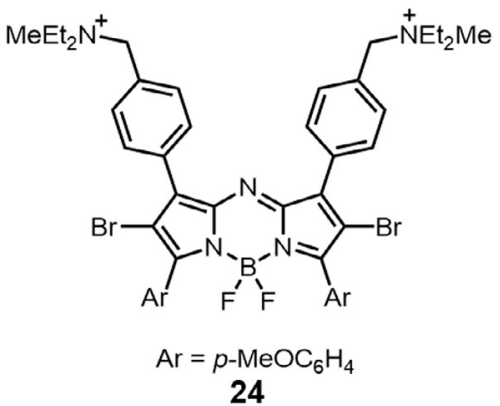

24

$$
\begin{gathered}
\Lambda_{\text {abs }}=521 \mathrm{~nm} \\
\kappa_{\text {ems }}=533 \mathrm{~nm} \\
\Phi \Delta=0.9 \text { fold of } \mathrm{MB}, \mathrm{ACN}
\end{gathered}
$$<smiles></smiles>

25

$$
\begin{gathered}
\Lambda_{\text {abs }}=546 \mathrm{~nm} \\
\Lambda_{\text {ems }}=590 \mathrm{~nm} \\
\Phi \Delta=0.20 \text { fold of RB, IPA }
\end{gathered}
$$<smiles></smiles>

26

$$
\begin{gathered}
\Lambda_{\text {abs }}=548 \mathrm{~nm} \\
\hat{\Lambda}_{\text {ems }}=595 \mathrm{~nm} \\
\Phi \Delta=0.08 \text { fold of RB, IPA }
\end{gathered}
$$

Fig. 8. Cationic BODIPY derivatives 24-26 for antimicrobial photodynamic therapy.

activity of compound $\mathbf{2 5}$ on Pseudomonas aeruginosa which is a widespread Gram-negative planktonic microorganism that has the ability to colonize and form biofilms [67]. Those biofilms can locate in many body parts such as lungs and diabetic wounds as well as in household and medical related surfaces. $P$. aeruginosa within the lower layers of biofilms exhibit slower metabolic activity and high resistance to antibiotics. Since APDT is a viable method where antibiotic treatment is inefficient, experiments with compound 25 showed decrement in the cell viability at even $2.5 \mu \mathrm{M}$ and prevention of regrowth due to photoinactivation. Control experiment resulted that the photosensitizer has dark toxicity in the higher concentrations $(80 \mu \mathrm{M})$; however, additional growth for $24 \mathrm{~h}$ fully overcome the intrinsic toxicity. Cationic photosensitizer structures can be adapted to potential medical applications like self-sterilizing surfaces and other equipment which need to have anti-infective feature.

$\mathrm{Li}$ and co-workers designed a polymer based macromolecular photosensitizer with antibacterial activity toward both Gramnegative and positive bacterial strains [68]. Introduction of the polymeric conjugation to photosensitizer overcomes the problems related to aggregation of BODIPY's hydrophobic structure in aqueous based biological media, enhancing the cytocompatibility. For that purpose, iodinated BODIPY derivative was copolymerized with 2-(dimethylamino)ethyl methacrylate and galactose. 2(Dimethylamino)ethyl methacrylate has cationic character in aqueous media so that it increases the binding efficiency to bacterial cell walls, on the other hand galactose unit decreases the cytotoxic and pro-inflammatory effects of positively charged polymer, increasing the macromolecules biocompatibility. The results indicated that the macromolecule disturbs the cell membrane of the $S$. aureus and $P$. aeruginosa bacteria leading the apoptotic cell death as shown in the SEM images in Fig. 9. Dark experiments have shown that the antibacterial activity of the macromolecules was poor compared with under light irradiation experiments.

Similar approach was demonstrated with the conjugation of porphyrin and BODIPY based photosensitizers to the cellulose fibers in order to form self-sterilizing, antibacterial paper sheets by Ghiladi and his co-workers [69]. An alternative targeting unit, 


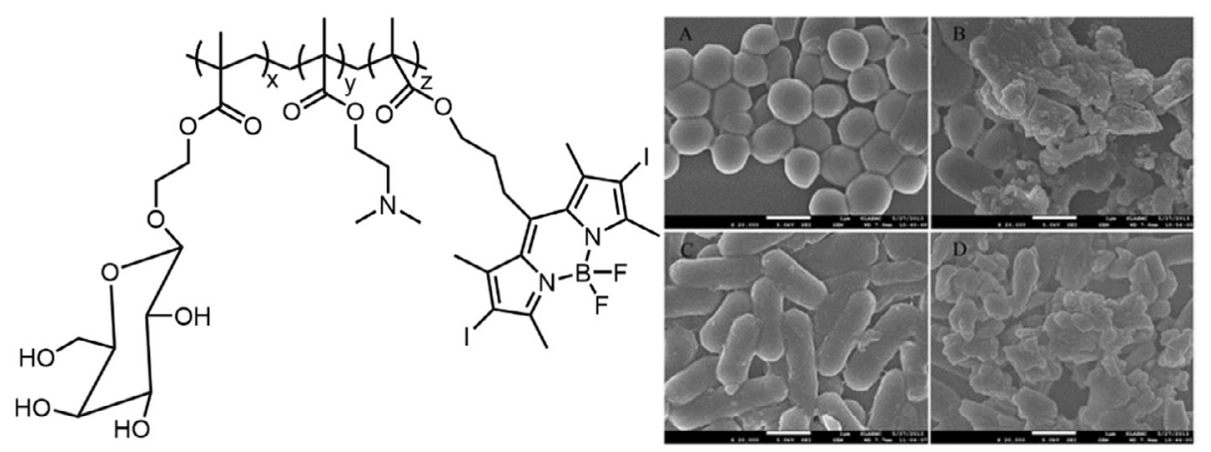

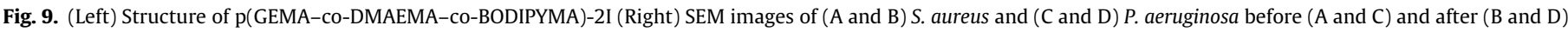
illumination. Adapted with permission from Ref. [68]. Copyright (c) 2015, Royal Society of Chemistry.
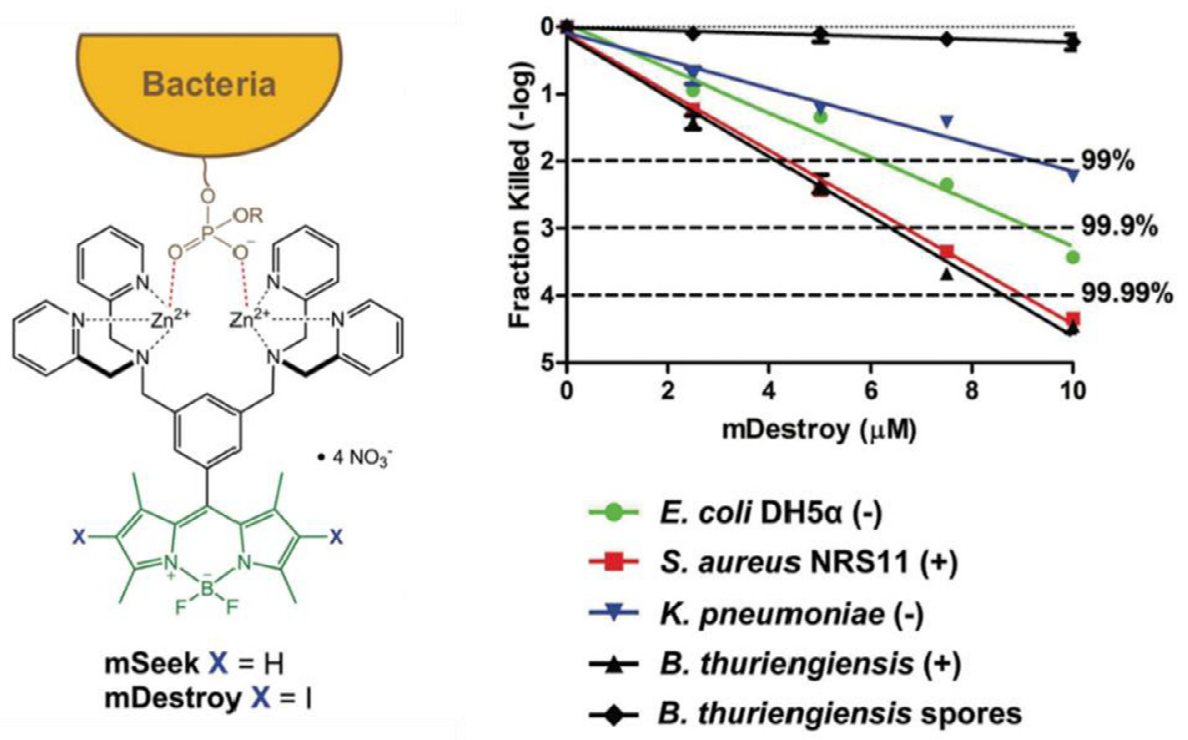

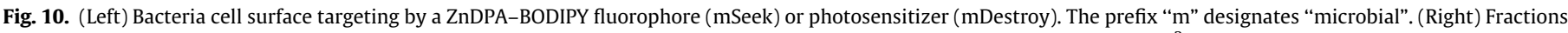

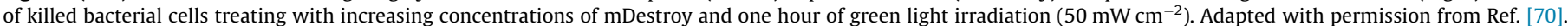
Copyright $\odot 2015$, Royal Society of Chemistry.

zinc(II)-dipicolylamine (ZnDPA), was utilized by Smith and coworkers in the place of cationic module for the targeted imaging and photodynamic action for eradicating pathogens as seen in Fig. 10. ZnDPA can coordinate with anionic groups like phosphate containing units on the bacterial cell membrane preferentially, whereas remain nearly inert to the neutral surface of healthy mammalian cells [70] (Fig. 11).mDestroy exhibited 99-99.99\% inactivation of four bacterial stains with selectivity in the presence of mammalian cells, besides mSeek, non-halogenated derivative of mDestroy, used for fluorescence imaging and detection with a CCD camera. As proposed in the aforementioned examples, photosensitization with BODIPY dye is an effective and selective options for the inactivation of pathogens on non-in vivo surface sterilization due to applicability in wide range of bacterial strains, which is independent of drug resistance.

\section{Modulation of photosensitization activity}

As described in previous sections, photodynamic therapy has highly desirable features in various applications in medicinal areas, including tumor diagnostics and treatments, or antibacterial/ antiviral activities. In clinical research, PDT has the ease of the localization of irradiation on desired area, thus it has great potential in cancer treatment techniques due to its non-invasive nature.
Still, some normal tissue and organ damaging causing edema around the treated tissues are reported [71-73]. Therefore besides the spacial selectivity, tumor-associated treatment strategies are required to decrease the side-effects. In the past decade, "turn off-on" photosensitizers which remain inert in bloodstream and normal tissues, but activated only when cancerous tissue or cells are encountered were reported [74-76]. Specific targeting to the cancerous tissues can be performed in many ways, because cancerous tissues have different chemical environments due to changes in metabolic/catabolic activities and over expression of some biological parameters and proteins. Novel designs regarding to the cancer selective PDT has been reported with BODIPY dyes via utilization of cancer specific moieties.

\subsection{Activation through $\mathrm{pH}$ responsive designs}

In order to especially target the tumor in the tissues or organs, one can take the advantage of characteristics of the microenvironment of tumor tissue. As studied on many cancerous cells, there is a difference in the concentrations of some ions or molecules between normal and cancer cells. As a consequence, those parameters are promising ways to access for the activation of therapeutic actions, including PDT. One of the parameters is the acidity of the media: the extracellular $\mathrm{pH}$ of the cancer cells is around 6.0, 
a
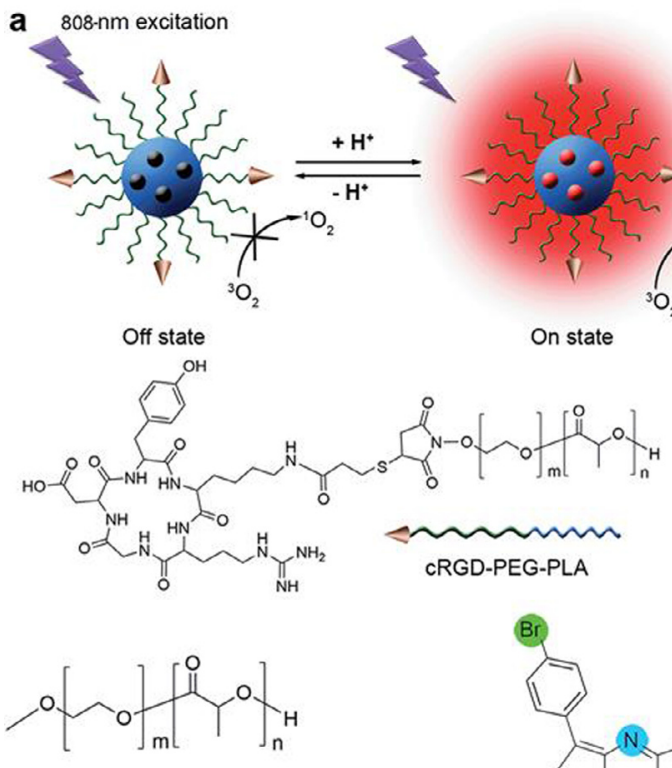

$\sim \sim \sim m$

MPEG-PLA

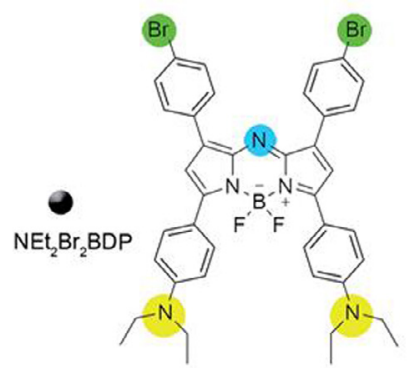

Fig. 11. Structures and $\mathrm{pH}$-activatable generation of fluorescence and ${ }^{1} \mathrm{O}_{2}$ by cRGD$\mathrm{NEt}_{2} \mathrm{Br}_{2} \mathrm{BDP}$ NP. Reprinted from Ref. [78]. Copyright (C) 2015, Royal Society of Chemistry.

whereas normal cells have slightly alkaline $\mathrm{pH}$ around at 7.4 [77]. Thus, many pH-activable systems including polymeric materials, nanocarriers besides photosensitizers are being developed. Ju and co-workers reported an aza-BODIPY derivative which is encapsulated in a cRDG-functionalized micelles which can be selectively taken up by $\alpha_{\mathrm{v}} \beta_{3}$ overexpressed tumor cells into lysosomes [78]. The usage of the polymer based agents for the encapsulation of photosensitizer are far more convenient than inorganic and metal based nanostructures due to biocompatibility and non-toxic degradation products in the body. In addition, the substituted $\mathrm{pH}$ responsive diethylaminophenyl group shows excellent switch-on selectivity in tumor imaging and photodynamic action in both in situ and real time experiments. The fluorescence and singlet oxygen quantum yield enhanced in the acidic media because of the fact that PET from the aniline group to BODIPY is no longer available. The system has also the advantage of absorbing in NIR region ( $\lambda \mathrm{abs}=822 \mathrm{~nm}$ ) which is essential for the penetration into the biological tissues.

Yan and co-workers, proposed similar design for $\mathrm{pH}$ activation of photodynamic therapy designing a polypeptide micelle which entrapped the photosensitizer, BODIPY- $\mathrm{Br}_{2}$, and has diisopropylamine group on the hydrophobic end regulating the on-off activation mechanism [79]. The core-shell structure of the nano micelles would degrade under acidic conditions ( $\mathrm{pH}$ 5.5) due to the protonation of the DIPEA groups conjugated to polypeptide side chains as shown in the Fig. 12, releasing the encapsulated BODIPY- $\mathrm{Br}_{2}$ into the desired area.

MTT assay with HepG2 (liver hepatocellular carcinoma) cells verified the selective photodynamic action in cancerous cells successfully under low energy density $\left(12 \mathrm{~J} / \mathrm{cm}^{2}\right)$ and low concentrations $(5.4 \mu \mathrm{M})$ with $45 \%$ higher mortality compared to the cells incubated under dark.

\subsection{Activation by biological thiols}

The intracellular glutathione concentration is another successful approach to the modulation of activatable PDT since glutathione (GSH) concentration in cancerous cells was reported up to 1000 -fold higher compared to normal cells [80-82]. Considering this tremendous difference in concentration of GSH between normal and cancer cells, a water-soluble BODIPY derivative was developed by Akkaya and co-workers which contains GSH-cleavable quencher moiety, so that "caged photosensitizer" can only generate cytotoxic singlet oxygen in GSH over-expressed medium [83]. While singlet oxygen generation capability is restored, fluorescence is also reinstated by the removal of the electron-sink quencher in $30 \mathrm{~min}$ with $80 \%$ yield, so that the progress of the cleavage reaction can be monitored. The behavior of the design was also validated by the cell culture experiments with human epithelial cancer cells (Huh7, MCF7, HCT116). It was proven that the photosensitizer is nontoxic and not capable of generation of singlet oxygen at its caged form in human fetal lung fibroblast cells (MRC-5) where GSH level is low, however with the uncaging in high GSH levels of cancer cells, photosensitization is active and causes the apoptosis resulting the death. For HCT116 cell lines, $\mathrm{IC}_{50}$ value under irradiation is $20.0 \mathrm{nM}$, on the other hand under dark, $\mathrm{IC}_{50}$ value is much higher $(4.38 \mu \mathrm{M})$, showing that the photosensitizer has comparably less dark toxicity. Another perspective to the GSH activation for PDT is to alter the properties of energy states of the photosensitizer to turn on-off the singlet oxygen

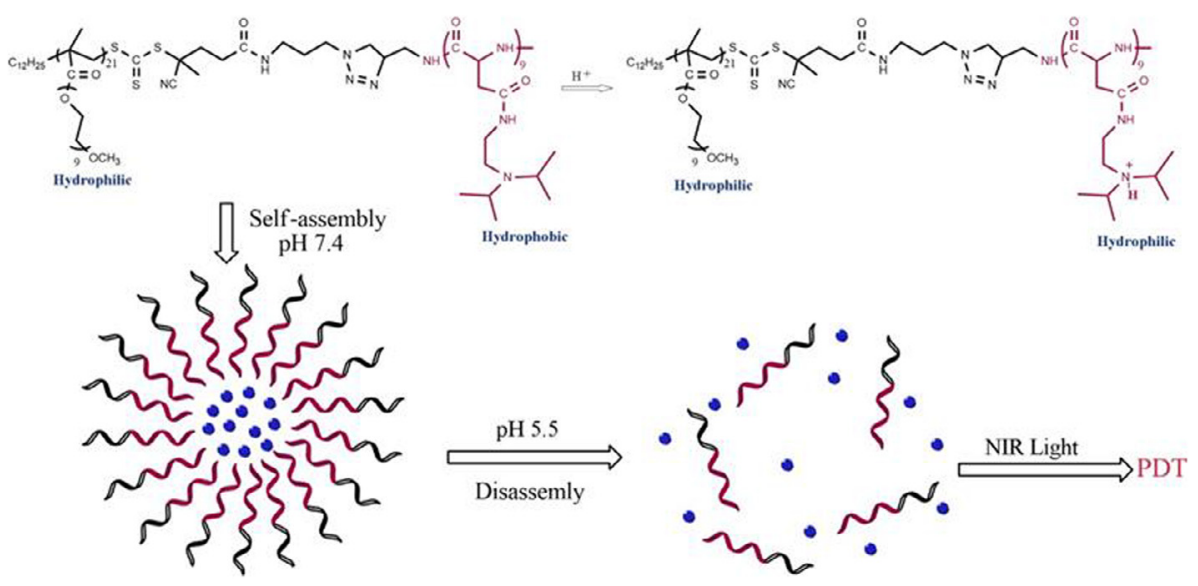

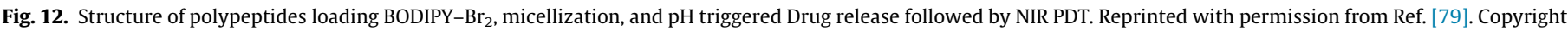
(c) 2016, American Chemical Society. 
production by reacting with GSH [31]. Proposed dissymmetric dimer of BODIPY dyes shown in Fig. 13, has nearly no photosensitization of molecular oxygen into singlet oxygen as a consequence of not having a degenerate set of HOMO and LUMO resulting lower intersystem crossing. The BODIPY dimer remains in its passive state until introduction into high-GSH level. GSH cuts the extension of conjugation and isolates the methylpyridinium group by either reduction or conjugate addition to the styryl double bond resulting the formation of the similarity and orthogonality which increases the ISC efficiency because dimeric BODIPY has a special electronic arrangement that similar subunits possesses a degenerate pair of HOMO and LUMO. Cell culture with HeLa cells studies also showed revealing results: photodynamic action of $\mathbf{2 8}$ causes the apoptotic death and HeLa cells have $38 \%$ survival rate upon the treatment of $\mathbf{2 8}\left(\mathrm{IC}_{50}: 280 \mathrm{ng} / \mathrm{mL}\right.$ for $\mathbf{2 8}, 32.6 \mathrm{ng} / \mathrm{mL}$ for $\mathbf{2 8 r}$ ), however normal NIH 3 T3 cells have $80 \%$ survival rate under same conditions. Also, no significant dark toxicity was observed without irradiation. All the finding showed that the design can stay passive even it absorbs the light, and regains photosensitization ability when cancerous tissue is encountered which has high concentrations of glutathione.

\subsection{Activation by enzymatic degradation}

Another promising attempts toward specific targeting is the utilization of enzyme-activated treatment of the cancerous tissues. Besides targeting the tumors, the on-site activated photosensitization was developed by Dong and his co-workers recently [84]. A diiodostyryl BODIPY substituted hyaluronic acid nanoparticles (DBHA-NPs, Fig. 14a) were introduced, which shows enhanced hydrophilicity and biocompatibility due to the presence of hyaluronic acid. The nanoparticles are composed of self-assembled aggregation of DBHA, which can disaggregate inside the lysosomes (hyaluronidase cause the enzymatic degradation of the HA polymer) and the singlet oxygen generation ability, under irradiation at $588 \mathrm{~nm}$, is restored as a consequence of disassembly. Besides its photodynamic efficiency, it has ability to regain fluorescence after disaggregation which makes it beneficial for diagnostic purposes. In vivo and in vitro experiment demonstrated that the design has excellent targeting ability to CD44 receptors which are overexpressed on the surface of HCT-116 cells without dark toxicity (Fig. 14b and c), resulting reduction of side effects in photodynamically activatable cancer therapy.

\subsection{Activation through ROS responsive design}

Utilization of activation with reactive oxygen species is an alternative way of targeting since cancer cells are generally under oxidative stress due to the increased metabolic activity resulting ROS generation. A two segment photosensitizer-trap which can be activated in an increased ROS was shown by Cosa recently
[85]. A naturally occurring antioxidant, chromanol ring of $\alpha$ tocopherol, which is an efficient scavenger of ROS, was modulated on brominated BODIPY core as photosensitizer. The trap moiety is both an efficient PET donor which quenches the singlet oxygen generation capability of BODIPY unit and a good physical ${ }^{1} \mathrm{O}_{2}$ quencher itself. Besides, when it reacts with ROS, PET is deactivated, and photosensitizer regains the singlet oxygen generation ability by around 40 -fold (Fig. 15). Since singlet oxygen is a ROS indeed, the activation of ${ }^{1} \mathrm{O}_{2}$ generation is an autocatalytic process, enhancing cytotoxic singlet oxygen generation by itself. The usefulness of this approach was demonstrated on Gram negative $E$. coli strain. One part of the cell lines were stressed with hydrogen peroxide which stimulates ROS production in Gram-negative cells and those cells showed an excessive drop in colony forming units compared to non-stressed healthy cells in the presence of compound and light irradiation. The PD-MBC (Photodynamic Minimum Bactericidal Concentration) value for the pre-activated compound by ROS was determined as $10 \mu \mathrm{M}$, and PD-MIC (Photodynamic Minimum Inhibitory Concentration) was reported as $10 \mu \mathrm{M}$ under light irradiation and $40 \mu \mathrm{M}$ in dark conditions for the same compound. This study is a successful example for ROS mediated activation of photodynamic therapy which can also amplify cytotoxic singlet oxygen generation by itself Fig. 16.

\subsection{Logic gates}

To enhance the tumor selectivity, one can take advantage of more than one characteristics of intercellular and intracellular chemical environment of cancerous tissues. For that purpose, Boolean logic, which is a key facet of computer programming, can also have significant potential in molecular systems which was introduced by de Silva in 1993 [86]. Molecular logic gates have been adapted to several examples including photodynamic therapy so that the photosensitizer would generate singlet oxygen as a response to two characteristic parameters together in the chemical environment. This system was first demonstrated with a BODIPY dye $\mathbf{3 0}$ in Akkaya group with a crown-ether-based PET modulator for $\mathrm{Na}^{+}$and pyridylethenyl module for $\mathrm{H}^{+}$input because both $\mathrm{Na}^{+}$ and $\mathrm{H}^{+}$concentrations are found significantly higher in cancer tissues than normal tissues [87]. When $\mathrm{Na}^{+}$concentration is high, PET is quenched and singlet oxygen generation is enhanced, on the other hand, in acidic medium, a red shift from $630 \mathrm{~nm}$ to $660 \mathrm{~nm}$ in absorption spectra is observed. Under irradiation at $660 \mathrm{~nm}$, the dye produces singlet oxygen most efficiently - about 6 -foldonly when pyridine groups are protonated in acidic media and PET is quenched in the presence of $\mathrm{Na}^{+}$ions, thus an AND logic gate is proposed where singlet oxygen is the output. This design was improved in Akkaya group later, integrating GSH and acidic response to form another AND logic gate [88]. Another aspect of thiol and $\mathrm{pH}$ activable photosensitization was demonstrated by $\mathrm{Ng}$ and Lo [89]. A halogenated BODIPY 31 derivative

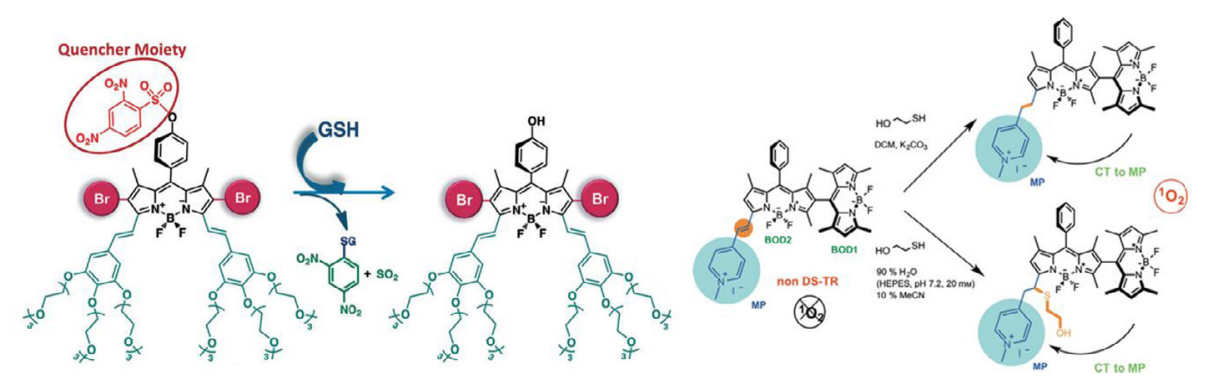

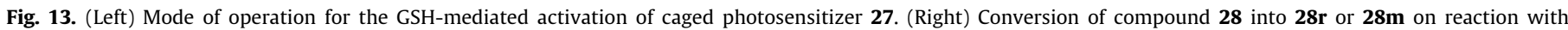

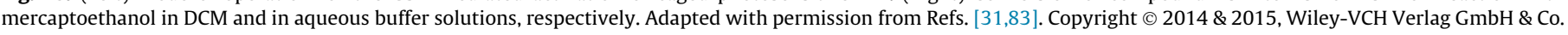
KGaA, Weinheim. 

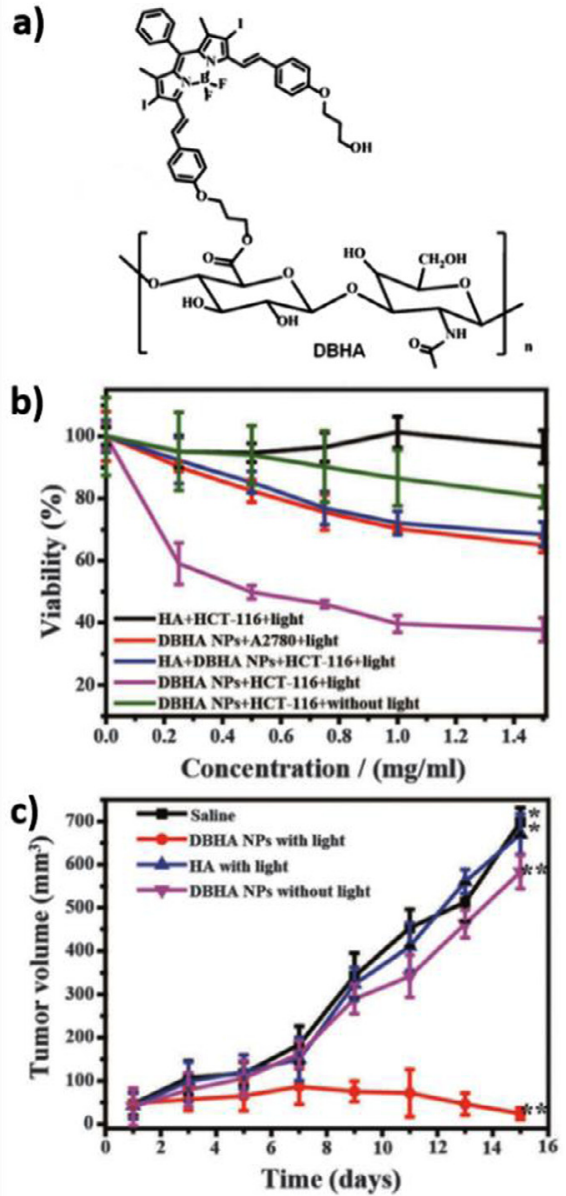

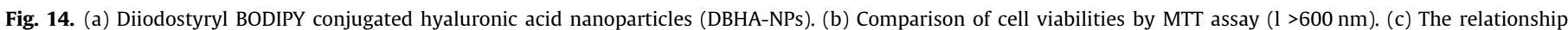

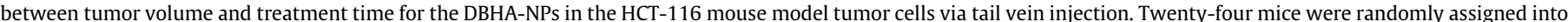

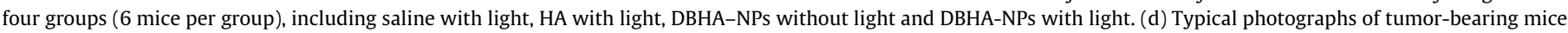
treated at different times. Adapted with permission from Ref. [84]. Copyright (c) 2016, Royal Society of Chemistry.

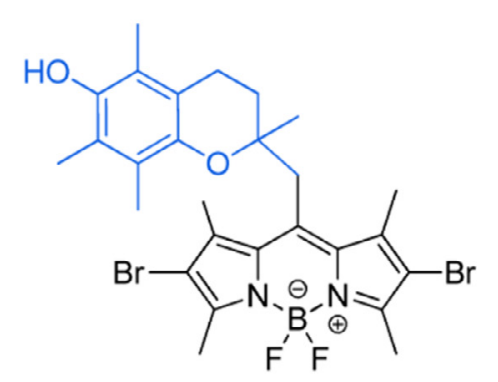

29

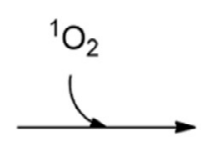

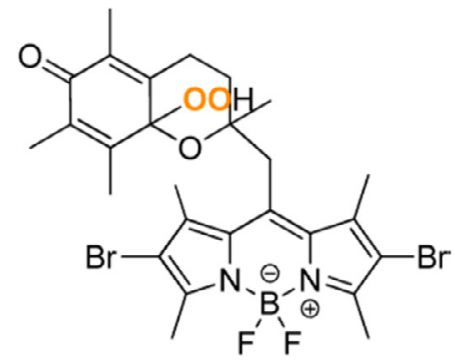

Fig. 15. Proposed Reaction of $\mathbf{2 9}$ with ${ }^{1} \mathrm{O}_{2}$. Reprinted with permission from Ref. [85]. Copyright @ 2016, American Chemical Society.

was functionalized with two ferrocenyl groups which are employed as quencher through PET mechanism. Ferrocenyl moieties are linked through a pH-cleavable ketal and a thiol-labile disulfide linkers so that the fluorescence and singlet oxygen generation efficiency will be enhanced by the coexistence of two-tumor associated parameters. In vitro results with MDF-7 breast cancer cells which are pretreated with dithiothreitol (DTT) were in agreement with the dual activation mechanism for the both fluorescence imaging and photodynamic activity of the design ( $\mathrm{IC}_{50}$ value of $140 \mathrm{nM}$ in $2 \mu \mathrm{M}$ DTT). Moreover, nude mice having a HT29 human colorectal carcinoma was treated with this compound and the restoration of fluorescence was observed inside the tumor within $9 \mathrm{~h}$, concluding that the cleavage of the two linkers by cutting the photoinduced electron transfer from ferrocenyl group to the dye was demonstrated which has great potential for theranostic and imaging of cancerous tissues.

\subsection{Control on PDT through PS delivery}

Besides the spacial selectivity of photosensitization, tumorassociated treatment can also be improved by introducing drug delivery systems for the theranostics of cancer. The incorporation 

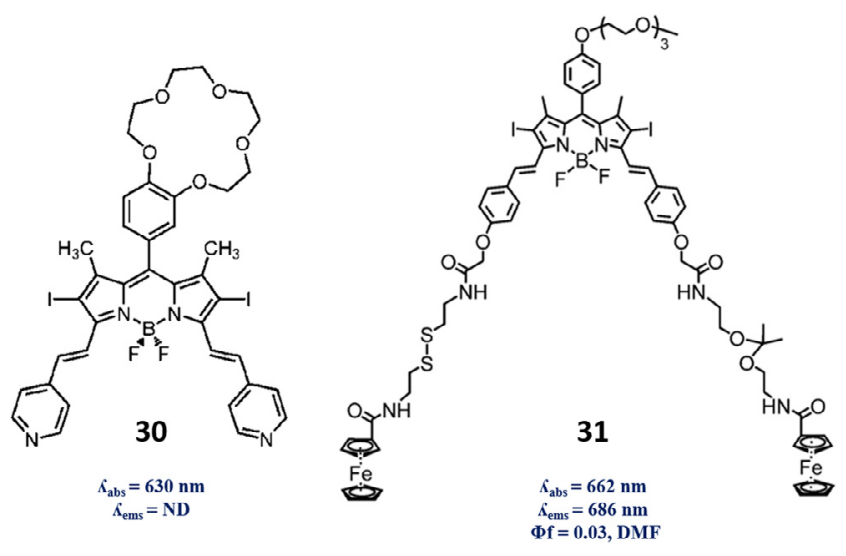

Fig. 16. Designs of the double-stimulus-responsive photosensitizers $\mathbf{3 0}$ and $\mathbf{3 1}$.

of the photosensitizer into nanomaterials enhances the permeability and retention effect along with biocompatibility thus therapeutic accuracy and efficiency increase [90,91]. Those carrier nanomaterials can be both organic based, such as polymers, liposomes etc. or inorganic structures like metallic composites [9294]. BODIPY derivatives substituted with n-decyloxyphenyl and pentadecyl groups which can embed themselves into micellar form and BODIPY chemisorbed on $\mathrm{NaYF}_{4}: \mathrm{Er}^{3+}, \mathrm{Yb}^{3+}$ up-conversion nanoparticles absorbing NIR region are both water soluble designs having desirable properties convenient for the biological media $[95,96]$. The enhanced permeability and retention (EPR) effect can be facilitated by incorporation of active targeting ligands onto the surface of nanostructures so that specific receptors on the cancerous cell membrane can be targeted selectively. Conventional targeting groups are based on galactose, folic acid, oligopeptides, and antibody derivatives $[97,98]$. For instance, HepG2 (liver hepatocellular carcinoma) cells overexpress ASGP-R galactose receptors so that it can be targeted by galactose functionalized amphiphilic copolymer micelles (PMAGP-POEGMA-PLys) as nanocarriers for
BODIPY, reported by Yan and co-workers [99]. POEGMA is the hydrophilic part of the polymer whereas PLys forms the hydrophobic core where hydrophobic BODIPY-PS is located (Fig. 17). PMAGP is the galactose containing targeting unit for the hepatoma carcinoma cells.

These nanostructures formed spherical micellar structure in aqueous media with $173 \mathrm{~nm}$ average size with the PS loading content about $2.5 \%$. Cell viability experiments were carried out for testing the dark toxicity and targeting ability of nanostructures. The nanostructures didn't exhibit dark toxicity in the absence of light irradiation and when they were exposed to light, cell viability decreased up to $50 \%$ for HepG2 cell lines while HeLa cells only decreased by $28 \%$ because endocytosis of the BODIPY containing micelles into the cells are more likely compared to HeLa cells which has lower ASGP-R galactose receptors. Similarly, Yin and co-workers designed mannose functionalized BODIPY nanoparticles for the selective targeting of lectin-type mannose-receptor overexpressing MDA-MB-231 breast cancer lines [100]. Long wavelength absorbing BODIPY derivative having three adamantane units assembled to a heptamannosylated $\beta$-cyclodextrin through supramolecular host-guest interactions forming spherical nanoparticles with $117 \mathrm{~nm}$ average size as given in Fig. 18.

MDA-MB-231 breast cancer lines were incubated with those nanoparticles, resulting photodynamic cellular damage and apoptotic cell death. On the other hand, the nanoparticles without mannose groups on the $\beta$-cyclodextrin didn't exhibit cellular damage under irradiation in same conditions. In vivo experiments also verified the selective targeted PDT for breast cancer cell lines without damaging normal cells and exhibiting very low dark toxicity. Usage of folate ligands for targeting purposes is an alternative convenient method for some cancer cell lines such as 4T1 breast cancer cells. The NIR absorbing dicarbazole functionalized BODIPY derivative was encapsulated by PLA-PEG-FA polymer to form a water soluble micellar structure [101]. TEM measurements indicated that the size of the dye loaded nanomicelles are around $14 \mathrm{~nm}$ which are quite smaller compared to related nanostructures. The tumor targeting ability were studied with HeLa cells in comparison with

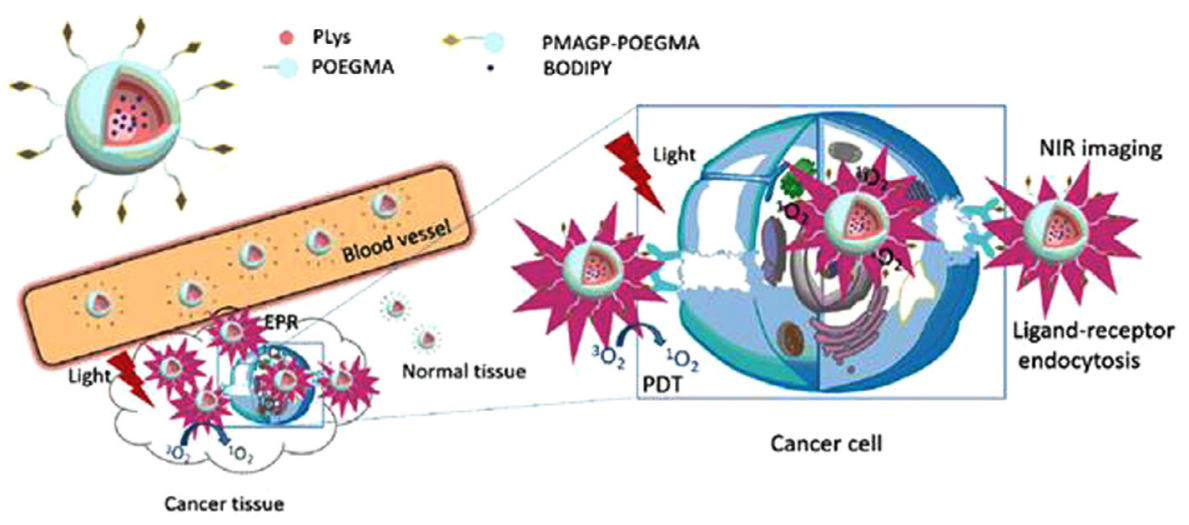<smiles>COc1ccc(-c2sc3cc4n(c3c2Br)[B-](F)(F)n2c-4cc3sc(-c4ccc(OC)cc4)c(Br)c32)cc1</smiles>

Fig. 17. Galactose-targeting and light-confined NIR imaging and photodynamic therapy on hepatoma cells with 32. Reprinted with permission from Ref. [99]. Copyright $\odot$ 2016, Royal Society of Chemistry. 

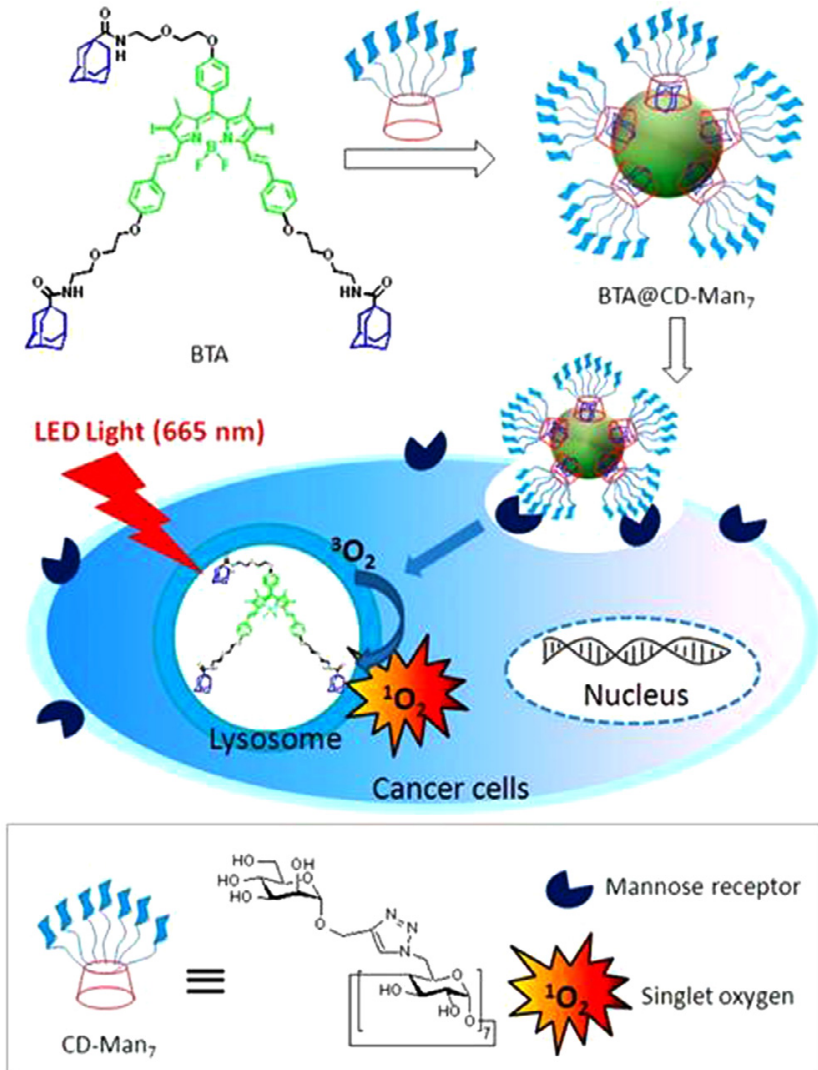

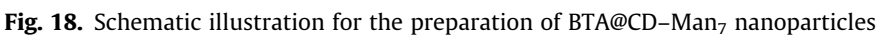
and their application for targeted PDT. Reprinted with permission from Ref. [100]. Copyright @ 2016, American Chemical Society.

healthy human skin epidermal Greengo cells. MTT assay and in vivo demonstration results verified the folate ligand improve the selectivity in cancer cells and no obvious organ dysfunction, dark toxicity, inflammation lesion abnormal results in serum analysis was observed. Those novel designs with the employment of nanoscale structures provide promising results for the platform for selective tumor photodynamic action for clinical applications.

\subsection{Control on PDT through subcellular localization}

Photodynamic therapy has been shown to operate in various mechanisms to destroy cancer cells, containing direct cell death among destruction of tumor vasculature and triggering immune response [45,102-105]. Even though each mechanism cooperates, direct cell death of tumor is beneficiary in terms of control on pho- todynamic effect. That is to say, cellular uptake can be utilized to effectively direct treatment on oxidative damage sensitive subcellular parts; organelles. For instance, it has been reported that nucleus is far more vulnerable to oxidative damage compared to plasma membrane $[106,107]$. Therefore, rational designs targeting oxidative damage sensitive subcellular organelles are far more likely to outperform.

Very first example of application of BODIPY dye $\mathbf{1 5}$ as a photosensitizer on $\mathrm{K} 562$ human erythroleukemia cells reported by Akkaya et al. revealed water soluble $\mathbf{1 5}$ decreases cell viability even under low fluence rate irradiation [43]. Following such phototherapeutic demonstration, unsymmetrical di-styryl BODIPY dyes 33-37 were analyzed in terms of photo-toxicity and subcellular localization [108]. Cellular uptake and photo-toxicity analysis of 33-37 on HT29 human colorectal carcinoma cells by intracellular fluorescence indicated greater uptake of $\mathbf{3 6}$ and 37, leading enhanced cytotoxicity with $\mathrm{IC}_{50}$ values around $15-17 \mathrm{nM}$. Detailed subcellular localization analysis showed localization of $\mathbf{3 7}$ on plasma membrane attributed to positively charged nature of the drug. On the other hand, $\mathbf{3 6}$ was found to localize on lysosome which isn't considered to be high priority target since other organelles localized photosensitizers outperform due to aggregation of dyes in lysosome (Fig. 19).

Endoplasmic reticulum has been reported to lead apoptotic cell death through caspase cascade upon being exposed to PDT. Compound 38, found to specifically localize on ER and lesser extent to mitochondria, outperformed in photo-cytotoxicity compared to its counterpart 39, which exhibited no subcellular localization, due to oxidative sensitivity of endoplasmic reticulum $\left(\mathrm{EC}_{50}\right.$ values for HeLa, A549 and MRC-5 of 4.7, 6.8, and $18.5 \mu \mathrm{M}$ without observable dark toxicity, respectively) [109]. In addition, Borggraeve and co-workers reported that BODIPY dye $\mathbf{4 0}$ and $\mathbf{4 1}$ co-localized in lysosome and endoplasmic reticulum of with T24 human urinary bladder carcinoma cell line; however, staining imaged of nuclear sequence localization peptide embedded $\mathbf{4 0}$, even though showed absence of $\mathbf{4 0}$ in nucleus, showed enhanced localization on ER compared to 41, explaining 2.6 times lower $\mathrm{IC}_{50}$ value of $\mathbf{4 0}$ compared to control structure 41 [110]. Aforementioned studies conducted with BODIPY dyes lead to similar results in terms of higher oxidative sensitivity of endoplasmic reticulum.

Instead of ER or nucleus, mitochondria regulating oxidative stress, metabolic energy and apoptotic machinery serves as a better target since mitochondrial integrity is essential for viability of cells. Compound $\mathbf{4 2}$ was prepared by Chakravarty and coworkers for a combinatory effect of PDT and chemotherapy such that mitochondria localized complex was expected to operate in such a way that release Pt from the complex is capable of inhibiting replication of mt-DNA (mitochondrial DNA) and BODIPY moiety embedded into the complex produces singlet oxygen for oxidative damage [111]. Further analysis on HaCaT human skin keratinocyte
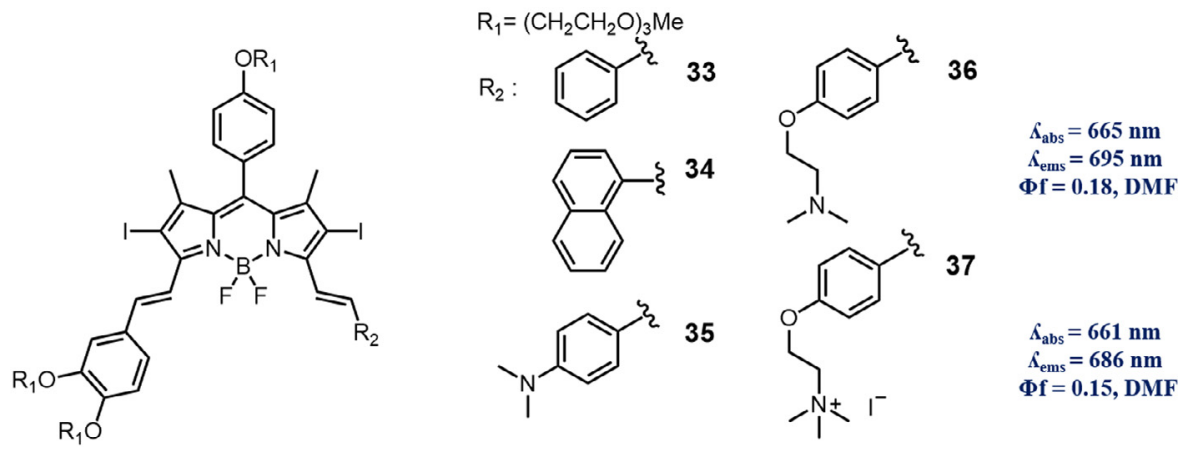

Fig. 19. Unsymmetrical di-styryl BODIPY derivatives 33-37. 


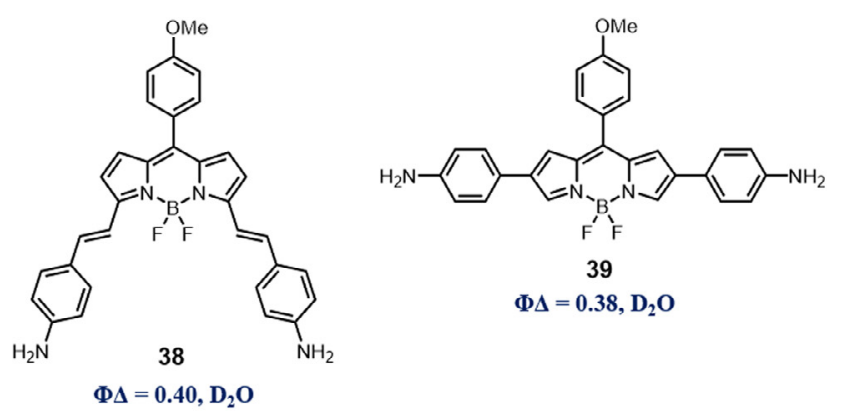

(n)
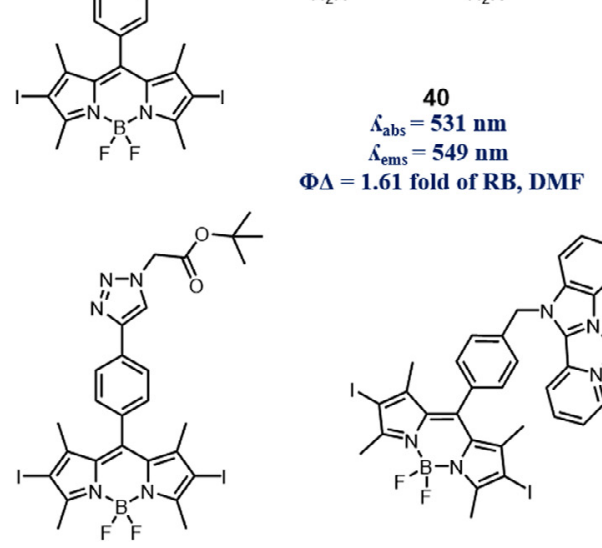

41

$\boldsymbol{K}_{\mathrm{abs}}=\mathbf{5 3 1} \mathrm{nm}$

$\hat{K}_{\mathrm{ems}}=\mathbf{5 4 9} \mathrm{nm}$

$\Phi \Delta=1.76$ fold of RB, DMF

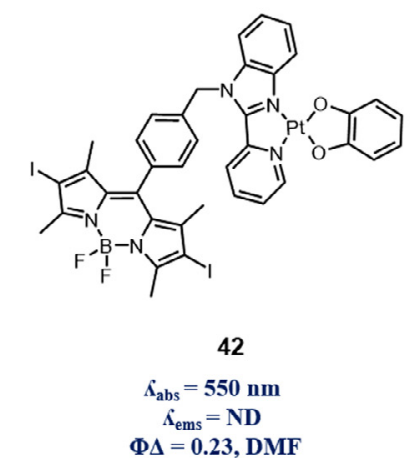

Fig. 20. BODIPY derivatives 38-42 designed for subcellular localization purposes.

cells indicated localization of BODIPY in mitochondria via confocal imaging as rationally designed. In another study, ternary copper(II) complex embedded with curcumin and BODIPY dye with similar working principle was shown to be preferentially localized in mitochondria of HeLa cancer cells, leading decrease in viability of cancer cells $\left(\mathrm{IC}_{50}\right.$ values of $3.8 \pm 0.2 \mu \mathrm{M}$ after PDT treatment and around $30 \mu \mathrm{M}$ under dark conditions) being comparable to first approved cancer drug Photofrin ${ }^{\circledR}$ [112]. That is to say, rational design of PDT agents with particular subcellular localization may enhance the control on phototherapeutic effect (Fig. 20).

\section{Photocatalysis by BODIPY dyes}

In the steadily developing research on photooxidation reactions, many catalyst species are reported in literature such as rubidium and iridium based photocatalysts [113-118]. Considering drawbacks of organometallic catalysts such as requirement of UV-light, high toxicity and cost, scientists tend to find new organic catalysts for oxidation reactions. 9,10-dicyanoanthracene, triphenylpyrylium tetrafluoroborate, n-methylquinolinium tetrafluoroborate as metal-free photocatalysts working in visible range gave promising results for that purpose [119-121]. Organic dyes, like BODIPY dyes, also play a significant role acting as photocatalysts instead of metal containing catalysts ascribed to having advantageous properties such as low toxicity, ease of functionalization and low cost. Photocatalytic efficiency of organic dyes are examined by Zeitler for photoredox transformations with various simple, inexpensive organic dyes such as rhodamine B, alizarin red S, perylene and xanthene derivatives [122]. With the great degree of functionalization feasibility, excellent thermal and photochemical stability, high singlet oxygen quantum yield and good solubility, BODIPY dyes are also possible candidates for photooxidation reactions, yet in this context only direct singlet oxygen involved catalytic systems are discussed.

\subsection{Photooxidation of sulfides}

Preparation of organic sulfoxides from sulfides are essential for organic synthesis not only for rudimentary research but also for multifarious applications, especially for biological and synthetic considerations [123-126]. Due to its significance, oxidation of sulfides has been drawing attention for many decades. Mild and high yielding conditions, which prevent side reactions including sulfone production have been desired and investigated in the synthetic and manufacturing manner. Herein, photocatalytic organic reactions introduce environmentally friendly, mild reactions with relatively high yields and lower production of by-products. Singlet oxygen forms the persulfoxide intermediate to form sulfoxide [127,128]. Xie first reported the photooxidation of sulfides, here thioanisole, with four BODIPY derivatives which are highly effective photocatalysts with 89-99\% conversion in methanol under visible light irradiation [129] (Fig. 21).

Then the results were improved by optimizing the reaction conditions and introducing four new BODIPY derivatives, concluding that the catalytic activity of BODIPY is highly dependent on singlet oxygen quantum yield and the protic solvent (methanol/ethanol) is beneficial to this photooxidation reaction [130]. Cui and He examined this catalytic reaction with ten various BODIPY derivatives using six thioanisole substrates [131]. Given BODIPY dyes in photocatalytic oxidation of sulfides, the ones which have higher singlet oxygen quantum yields: orthogonal dimeric BODIPY and halogenated derivatives, demonstrated higher oxidation yields, supporting the findings by Jing. Indeed, this work proved that BODIPY dyes are successful alternatives for known photosensitizers such as Nile Red and Ru-based organometallic catalysis.

\subsection{Photooxidation of dihydroxynaphthalenes}

Singlet oxygen can also form endoperoxide intermediate with hydroxynaphthalene substrates yielding dihydroxynaphthalene (DHN) derivatives. One studied example is juglone which is a chemical used as herbicides or coloring agent in cosmetics, food industry and cloth (Fig. 22).

BODIPY derivatives conjugated with 2-(2-hydroxyphenyl)benzo thiazole and benzoxazole subunits for excited state intramolecular proton transfer (ESIPT) were prepared by Zhao and co-workers and used as ${ }^{1} \mathrm{O}_{2}$ photosensitizer for the photocatalytic oxidation of 1,4dihydroxynaphtalene [132]. These photosensitizers exhibited more efficiency compared to the traditional transition metal complexes, like ([Ir(ppy) $)_{2}($ phen $\left.\left.)\right]\left[\mathrm{PF}_{6}\right]\right)$. Zhao and co-workers also prepared iodo-BODIPY derivatives with strong absorption of visible light and efficient long-lived triplet excited state for ${ }^{1} \mathrm{O}_{2}$ mediated dihydroxynaphthalenes photooxidation to produce specific naphthalenedione derivatives which are further used for production of some anticancer and antibiotic reagents [133]. The yields were relatively lower (73-87\%), however the reactions took place in just 30 min which is considerably fast compared to other photooxidation reactions. Mechanism of those reactions rely on oxidization of naphthol by singlet oxygen $\left({ }^{1} \mathrm{O}_{2}\right)$ which is produced by 
a)<smiles></smiles>

43

$$
\begin{gathered}
\Lambda_{\mathrm{abs}}=495 \mathrm{~nm} \\
\Lambda_{\mathrm{ems}}=511 \mathrm{~nm} \\
\text { EtOH }
\end{gathered}
$$

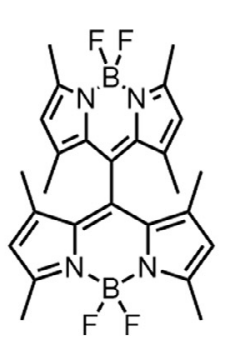

47

$$
\Lambda_{\text {abs }}=515 \mathrm{~nm}
$$$$
\hat{\Lambda}_{\mathrm{ems}}=606 \mathrm{~nm}
$$
$\Phi \Delta=0.46, \mathrm{CH}_{2} \mathrm{Cl}_{2}$<smiles></smiles>

\section{4}

$$
\begin{gathered}
\hat{\Lambda}_{\mathrm{abs}}=497 \mathrm{~nm} \\
\hat{\Lambda}_{\mathrm{ems}}=\mathbf{5 0 7} \mathrm{nm} \\
\text { ACN }
\end{gathered}
$$<smiles></smiles>

45

$$
\begin{gathered}
\hat{\Lambda}_{\mathrm{abs}}=494 \mathrm{~nm} \\
\hat{\Lambda}_{\mathrm{ems}}=516 \mathrm{~nm} \\
\mathrm{CH}_{2} \mathrm{Cl}_{2}
\end{gathered}
$$<smiles></smiles>

48<smiles>CC1=CC(C)=[N+]2B(F)n3c(C)cc(C)c3C(c3ccc(N(c4ccccc4)c4ccccc4)cc3)=C12</smiles>

\section{6}<smiles></smiles>

49

b)<smiles>CSc1ccccc1</smiles>

Fig. 21. BODIPY derivatives $\mathbf{4 3 - 4 9}$ for the oxidation of sulfides.

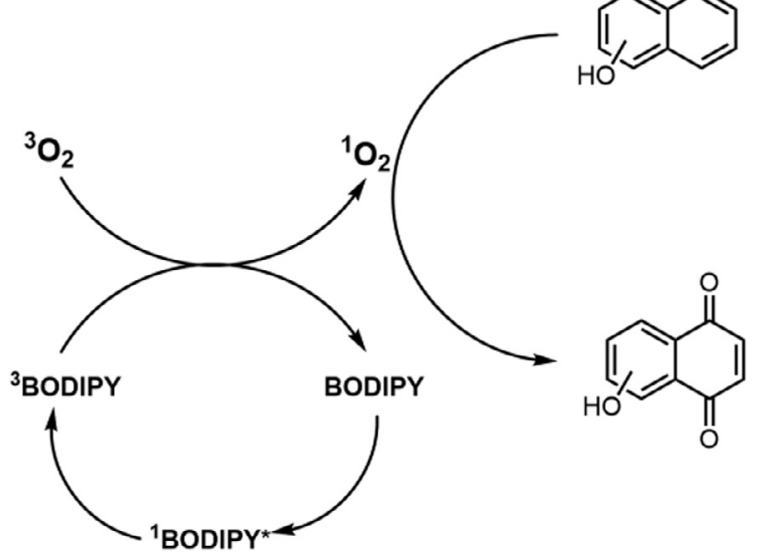

Fig. 22. Photooxidation of $\mathrm{DHN}$ with a triplet photosensitizer; BDP $=$ BODIPY compounds.

photosensitizing of the triplet oxygen $\left({ }^{3} \mathrm{O}_{2}\right)$ by the photosensitizers, BODIPY. Since, the aza-BODIPY derivatives showed high singlet oxygen generation capability, Ramaiah and co-workers synthesized halogenated aza-BODIPY dyes and explored their use as photooxygenation catalysts for 1-naphthol oxidation into juglone [134]. The highest yield was achieved under focused sunlight to yield $100 \%$ conversion in $30 \mathrm{~min}$. For comparison, the traditional photosensitizers, tetraphenylporphyrin, rose bengal and methylene blue, were used. Core and peripheral halogenated azaBODIPY derivative showed higher conversion efficiency than the known photosensitizers, despite having comparable singletoxygen efficiencies, because aza-BODIPY has better photostability under the irradiation conditions (more than $8 \mathrm{~h}$ ).

\subsection{Photocatalytic synthesis of 2-aryl benzothiazole}

Singlet oxygen generation capacity of BODIPY type dyes makes them highly desirable also for a photocatalyzed oxidative condensation reaction to produce 2-aryl benzothiazoles, which play crucial roles in various applications, containing biologically active products, drugs, fibers, plastics, liquid crystals, off-color materials along with several others. Zhou and Yang has reported that BODIPY 50 can enable the condensation reaction between amines and 2-aminothiophenol via production of singlet oxygen to produce 2aryl benzothiazole [135]. Reaction pursues oxidation of amine with singlet oxygen prior to condensation of intermediate with 2-aminothiophenol. In the follow up study, Yang et al. reported that BODIPY polymer, prepared from BODIPY $\mathbf{5 1}$ and 1-4-phenylenebisboronic acid through Suzuki coupling, loaded on silica powder showed enhanced photocatalytic activity and thermal 

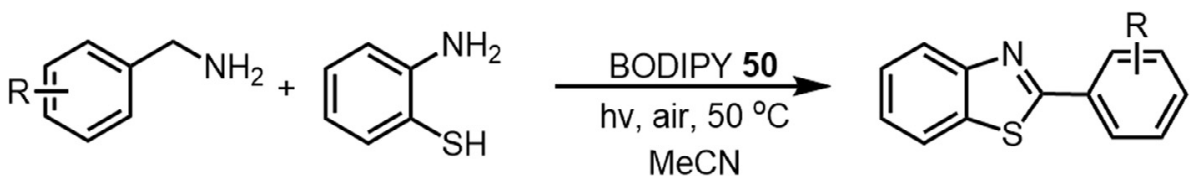

Fig. 23. General synthesis reaction of 2-aryl benzothiazole derivatives catalyzed by BODIPY $\mathbf{5 0 .}$
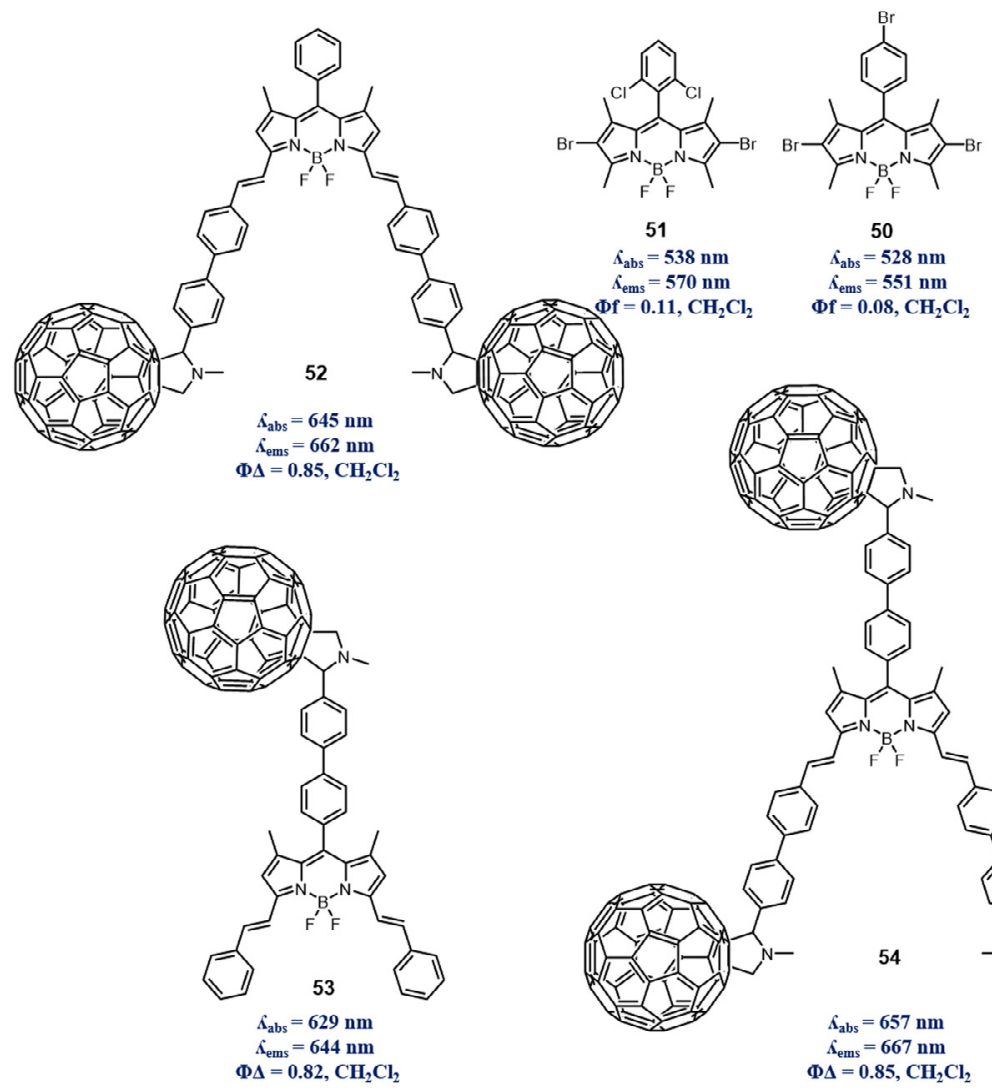

Fig. 24. Dyads 52, 53 and 54 along with BODIPY derivatives $\mathbf{5 0}$ and $\mathbf{5 1}$ for 2-aryl benzothiazole synthesis.

and photo stability along with improved dispersibility in the condensation reaction yielding 2-aryl benzothiazoles [136] (Fig. 23).

\subsection{BODIPY-fullerene dyads in photooxidation}

BODIPY dyes have been manipulated in several ways for photocatalytic applications, one of which is formation of dyads with fullerene. In this type of light harvesting designs, BODIPY derivatives are utilized as light harvesting antenna, and fullerene structure is designed as spin converter. This relies on the fact that fullerene structure has low-lying singlet excited state, enabling the energy transfer from chromophore to fullerene. Intramolecular energy transfer eventually populates $\mathrm{S} 1$ state of $\mathrm{C} 60$, resulting population of T1 state of C60 due to intrinsic ISC property of fullerene unit; however, this doesn't necessarily mean that triplet state localizes on fullerene unit. In contrast, due low lying triplet excited state of BODIPY unit compared to fullerene lead triplet energy transfer toward BODIPY unit. Zhao and co-workers reported BODIPY-fullerene dyads 52, 53 and $\mathbf{5 4}$ based on "ping-pong" energy transfer [137] (Fig. 24).

Application of these dyads on oxidation of 1,5-dihydroxy naphthalene to form juglone demonstrated their enhanced singlet oxygen production capability. Zhao and co-workers also reported
BODIPY-fullerene triads and tetrads which contain two and three antennas with varying absorption wavelengths, indicating broadband absorption nature of such designs [138]. Based on similar energy transfer processes, triads and tetrads were found to be even more effective in singlet oxygen production as observed in photocatalytic oxidation of DHN compared to TPP and MB. Therefore, without using heavy atoms, rationally designed energy harvesting systems can produce singlet oxygen for photocatalytic purposes.

\section{Conclusion}

BODIPY dyes are highly versatile, and a bewildering number of applications with BODIPY dyes playing essential roles were reported. As presented in this review, BODIPY dyes are very amenable to structural modifications making them viable alternatives for porphyrins and phthalocyanines as photosensitizer. It is also noteworthy that in recent years, most creative works on photosensitizers were based BODIPY dyes, most likely because of their synthetic and photochemical versatility. It is possible to precisely finetune the photosensitization characteristics of BODIPY derivatives. We are confident that future advances in photodynamic therapy, and photosensitizer based chemistry in general, will benefit tremendously for this upsurge of interest in BODIPY chemistry. 


\section{Acknowledgments}

The authors acknowledge support from Bilkent University. A.T. is also grateful for a scholarship from TUBITAK (BIDEB-2210 E).

\section{Appendix A. Supplementary data}

Supplementary data associated with this article can be found, in the online version, at https://doi.org/10.1016/j.ccr.2017.09.029.

\section{References}

[1] N.C. Zeitouni, A.R. Oseroff, S. Shieh, Mol. Immunol. 39 (2003) 1133-1136.

[2] Z. Huang, Technol. Cancer Res. Treat. 4 (2005) 283-293.

[3] D.E. Dolmans, D. Fukumura, R.K. Jain, Nat. Rev. Cancer 3 (2003) 380-387.

[4] S.B. Brown, E.A. Brown, I. Walker, Lancet Oncol. 5 (2004) 497-508.

[5] T.J. Dougherty, G.B. Grindey, R. Fiel, K.R. Weishaupt, D.G. Boyle, J. Natl Cancer Inst. 55 (1975) 115-121.

[6] J.F. Kelly, M.E. Snell, J. Urol. 115 (1976) 150-151.

[7] Y. Hayata, H. Kato, C. Konaka, J. Ono, N. Takizawa, Chest 81 (1982) 269-277.

[8] J.S. McCaughan Jr., W. Hicks, L. Laufman, E. May, R. Roach, Cancer 54 (1984) 2905-2910.

[9] Y. Hayata, H. Kato, H. Okitsu, M. Kawaguchi, C. Konaka, Semin. Surg. Oncol. 1 (1985) $1-11$.

[10] J.S. Hill, A.H. Kaye, W.H. Sawyer, G. Morstyn, P.D. Megison, S.S. Stylli, Neurosurgery 26 (1990) 248-254.

[11] E.A. Popovic, A.H. Kaye, J.S. Hill, J. Clin. Laser Med. Surg. 14 (1996) 251-261.

[12] M.A. Rosenthal, B. Kavar, J.S. Hill, D.J. Morgan, R.L. Nation, S.S. Stylli, R.L. Basser, S. Uren, H. Geldard, M.D. Green, S.B. Kahl, A.H. Kaye, J. Clin. Oncol. 19 (2001) 519-524.

[13] C.J. Gomer, D.R. Doiron, J.V. Jester, B.C. Szirth, A.L. Murphree, Cancer Res. 43 (1983) 721-727.

[14] I. Favilla, M.L. Favilla, A.D. Gosbell, W.R. Barry, P. Ellims, J.S. Hill, J.R. Byrne Melanoma Res. 5 (1995) 355-364.

[15] I.M. Landau, B. Steen, S. Seregard, Acta Ophthalmol. Scand. 80 (2002) 531 536.

[16] T.J. Dougherty, G. Lawrence, J.H. Kaufman, D. Boyle, K.R. Weishaupt, A. Goldfarb, J. Natl Cancer Inst. 62 (1979) 231-237.

[17] T.S. Mang, R. Allison, G. Hewson, W. Snider, R. Moskowitz, Cancer J, Sci. Am. 4 (1998) 378-384.

[18] A. Dimofte, T.C. Zhu, S.M. Hahn, R.A. Lustig, Lasers Surg. Med. 31 (2002) $305-$ 312.

[19] V.G. Schweitzer, Otolaryngol. Head Neck Surg. 102 (1990) 225-232.

[20] M.A. Biel, Laryngoscope 108 (1998) 1259-1268.

[21] S.G. Bown, A.Z. Rogowska, D.E. Whitelaw, W.R. Lees, L.B. Lovat, P. Ripley, L. Jones, P. Wyld, A. Gillams, A.W. Hatfield, Gut 50 (2002) 549-557.

[22] R. Hornung, Curr. Drug Targets Immune Endocr. Metabol. Disord. 1 (2001) $165-177$.

[23] M.K. Fehr, R. Hornung, V.A. Schwarz, R. Simeon, U. Haller, P. Wyss, Gynecol. Oncol. 80 (2001) 62-66.

[24] A. Kamkaew, S.H. Lim, H.B. Lee, L.V. Kiew, L.Y. Chung, K. Burgess, Chem. Soc. Rev. 42 (2013) 77-88.

[25] A. Treibs, F.-H. Kreuzer, Liebigs Ann. Chem. 718 (1968) 208-223.

[26] A. Gorman, J. Killoran, C. O'Shea, T. Kenna, W.M. Gallagher, D.F. O'Shea, J. Am. Chem. Soc. 126 (2004) 10619-10631.

[27] T. Yogo, Y. Urano, Y. Ishitsuka, F. Maniwa, T. Nagano, J. Am. Chem. Soc. 127 (2005) 12162-12163.

[28] Y. Cakmak, S. Kolemen, S. Duman, Y. Dede, Y. Dolen, B. Kilic, Z. Kostereli, L.T. Yildirim, A.L. Dogan, D. Guc, E.U. Akkaya, Angew. Chem. Int. Ed. Engl. 50 (2011) (1941) 11937-11941.

[29] S. Duman, Y. Cakmak, S. Kolemen, E.U. Akkaya, Y. Dede, J. Org. Chem. 77 (2012) 4516-4527.

[30] X.F. Zhang, X. Yang, J. Phys. Chem. B 117 (2013) 9050-9055.

[31] S. Kolemen, M. Isik, G.M. Kim, D. Kim, H. Geng, M. Buyuktemiz, T. Karatas, X.F. Zhang, Y. Dede, J. Yoon, E.U. Akkaya, Angew. Chem. Int. Ed. Engl. 54 (2015) 5340-5344.

[32] S.D. Topel, G.T. Cin, E.U. Akkaya, Chem. Commun. (Camb.) 50 (2014) 88968899.

[33] B. Ventura, G. Marconi, M. Bröring, R. Krüger, L. Flamigni, New J. Chem. 33 (2009) 428-438.

[34] T. Ozdemir, J.L. Bila, F. Sozmen, L.T. Yildirim, E.U. Akkaya, Org. Lett. 18 (2016) 4821-4823.

[35] Z. Feng, L. Jiao, Y. Feng, C. Yu, N. Chen, Y. Wei, X. Mu, E. Hao, J. Org. Chem. 81 (2016) 6281-6291.

[36] E. Dai, W. Pang, X.F. Zhang, X. Yang, T. Jiang, P. Zhang, C. Yu, E. Hao, Y. Wei, X. Mu, L. Jiao, Chem. Asian J. 10 (2015) 1327-1334.

[37] S.G. Awuah, S.K. Das, F. D’Souza, Y. You, Chem. Asian J. 8 (2013) 3123-3132.

[38] S.G. Awuah, J. Polreis, V. Biradar, Y. You, Org. Lett. 13 (2011) 3884-3887.

[39] Y. Yang, Q. Guo, H. Chen, Z. Zhou, Z. Guo, Z. Shen, Chem. Commun. (Camb.) 49 (2013) 3940-3942.

[40] K. Rurack, M. Kollmannsberger, J. Daub, New J. Chem. 25 (2001) 289-292.

[41] Z. Dost, S. Atilgan, E.U. Akkaya, Tetrahedron 62 (2006) 8484-8488.
[42] O. Buyukcakir, O.A. Bozdemir, S. Kolemen, S. Erbas, E.U. Akkaya, Org. Lett. 11 (2009) 4644-4647.

[43] S. Atilgan, Z. Ekmekci, A.L. Dogan, D. Guc, E.U. Akkaya, Chem. Commun. (Camb.) (2006) 4398-4400.

[44] X.-D. Jiang, D. Xi, B. Le Guennic, J. Guan, D. Jacquemin, J. Guan, L.-J. Xiao, Tetrahedron 71 (2015) 7676-7680.

[45] P. Agostinis, K. Berg, K.A. Cengel, T.H. Foster, A.W. Girotti, S.O. Gollnick, S.M. Hahn, M.R. Hamblin, A. Juzeniene, D. Kessel, M. Korbelik, J. Moan, P. Mroz, D. Nowis, J. Piette, B.C. Wilson, J. Golab, CA Cancer J. Clin. 61 (2011) 250-281.

[46] J. Wang, Y. Hou, W. Lei, Q. Zhou, C. Li, B. Zhang, X. Wang, ChemPhysChem 13 (2012) 2739-2747.

[47] A. Fraix, M. Blangetti, S. Guglielmo, L. Lazzarato, N. Marino, V. Cardile, A.C. Graziano, I. Manet, R. Fruttero, A. Gasco, S. Sortino, ChemMedChem 11 (2016) $1371-1379$.

[48] Z. Xiao, S. Halls, D. Dickey, J. Tulip, R.B. Moore, Clin. Cancer Res. 13 (2007) 7496-7505.

[49] L. Yang, Y. Wei, D. Xing, Q. Chen, Lasers Surg. Med. 42 (2010) 671-679.

[50] S.L. Gibson, K.R. VanDerMeid, R.S. Murant, R.F. Raubertas, R. Hilf, Cancer Res, 50 (1990) 7236-7241.

[51] I.S. Turan, D. Yildiz, A. Turksoy, G. Gunaydin, E.U. Akkaya, Angew. Chem. Int. Ed. Engl. 55 (2016) 2875-2878.

[52] B.C. Wilson, W.P. Jeeves, D.M. Lowe, Photochem. Photobiol. 42 (1985) 153162.

[53] A.P. Castano, T.N. Demidova, M.R. Hamblin, Photodiagnosis Photodyn. Ther. 1 (2004) 279-293.

[54] H. He, S. Ji, Y. He, A. Zhu, Y. Zou, Y. Deng, H. Ke, H. Yang, Y. Zhao, Z. Guo, H. Chen, Adv. Mater. 29 (2017).

[55] Q. Tang, W. Si, C. Huang, K. Ding, W. Huang, P. Chen, Q. Zhang, X. Dong, J. Mater. Chem. B 5 (2017) 1566-1573.

[56] L.B. Meng, W. Zhang, D. Li, Y. Li, X.Y. Hu, L. Wang, G. Li, Chem. Commun. (Camb.) 51 (2015) 14381-14384.

[57] Z. Ruan, L. Liu, W. Jiang, S. Li, Y. Wang, L. Yan, Biomater. Sci. 5 (2017) 313-321.

[58] S. Erbas-Cakmak, E.U. Akkaya, Org. Lett. 16 (2014) 2946-2949.

[59] J. Zhao, L. Huang, X. Cui, S. Li, H. Wu, J. Mater. Chem. B 3 (2015) 9194-9211.

[60] R.L. Watley, S.G. Awuah, M. Bio, R. Cantu, H.B. Gobeze, V.N. Nesterov, S.K. Das, F. D'Souza, Y. You, Chem. Asian J. 10 (2015) 1335-1343.

[61] G.P. Tegos, M. Anbe, C. Yang, T.N. Demidova, M. Satti, P. Mroz, S. Janjua, F. Gad, M.R. Hamblin, Antimicrob. Agents Chemother. 50 (2006) 1402-1410.

[62] Y.Y. Huang, T. Balasubramanian, E. Yang, D. Luo, J.R. Diers, D.F. Bocian, J.S. Lindsey, D. Holten, M.R. Hamblin, ChemMedChem 7 (2012) 2155-2167.

[63] T. Dai, Y.Y. Huang, M.R. Hamblin, Photodiagnosis Photodyn. Ther. 6 (2009) $170-188$.

[64] D.O. Frimannsson, M. Grossi, J. Murtagh, F. Paradisi, D.F. O’Shea, J. Med. Chem. 53 (2010) 7337-7343.

[65] E. Caruso, S. Banfi, P. Barbieri, B. Leva, V.T. Orlandi, J. Photochem. Photobiol., B 114 (2012) 44-51.

[66] B.L. Carpenter, X. Situ, F. Scholle, J. Bartelmess, W.W. Weare, R.A. Ghiladi, Molecules 20 (2015) 10604-10621.

[67] V.T. Orlandi, M. Rybtke, E. Caruso, S. Banfi, T. Tolker-Nielsen, P. Barbieri, Biofouling 30 (2014) 883-891.

[68] Z. Lu, X. Zhang, Y. Zhao, Y. Xue, T. Zhai, Z. Wu, C. Li, Polym. Chem. 6 (2015) 302-310.

[69] B.L. Carpenter, F. Scholle, H. Sadeghifar, A.J. Francis, J. Boltersdorf, W.W. Weare, D.S. Argyropoulos, P.A. Maggard, R.A. Ghiladi, Biomacromolecules 16 (2015) 2482-2492.

[70] D.R. Rice, H. Gan, B.D. Smith, Photochem. Photobiol. Sci. 14 (2015) $1271-$ 1281.

[71] J. Golab, D. Olszewska, P. Mroz, K. Kozar, R. Kaminski, A. Jalili, M. Jakobisiak, Clin. Cancer Res. 8 (2002) 1265-1270.

[72] M.S. Mathews, D. Chighvinadze, H.M. Gach, F.A. Uzal, S.J. Madsen, H. Hirschberg, Lasers Surg. Med. 43 (2011) 892-900.

[73] H. Hirschberg, F.A. Uzal, D. Chighvinadze, M.J. Zhang, Q. Peng, S.J. Madsen, Lasers Surg. Med. 40 (2008) 535-542.

[74] S.O. McDonnell, M.J. Hall, L.T. Allen, A. Byrne, W.M. Gallagher, D.F. O’Shea, J. Am. Chem. Soc. 127 (2005) 16360-16361.

[75] T. Yogo, Y. Urano, A. Mizushima, H. Sunahara, T. Inoue, K. Hirose, M. Iino, K. Kikuchi, T. Nagano, Proc. Natl. Acad. Sci. U.S.A. 105 (2008) 28-32.

[76] J. Chen, K. Stefflova, M.J. Niedre, B.C. Wilson, B. Chance, J.D. Glickson, G. Zheng, J. Am. Chem. Soc. 126 (2004) 11450-11451.

[77] R.A. Gatenby, R.J. Gillies, Nat. Rev. Cancer 4 (2004) 891-899.

[78] J. Tian, J. Zhou, Z. Shen, L. Ding, J.-S. Yu, H. Ju, Chem. Sci. 6 (2015) 5969-5977.

[79] L. Liu, L. Fu, T. Jing, Z. Ruan, L. Yan, A.C.S. Appl, Mater. Interfaces 8 (2016) 8980-8990.

[80] Z.B. Zheng, G. Zhu, H. Tak, E. Joseph, J.L. Eiseman, D.J. Creighton, Bioconjug, Chem. 16 (2005) 598-607.

[81] R.A. Cairns, I.S. Harris, T.W. Mak, Nat. Rev. Cancer 11 (2011) 85-95.

[82] C.C. Yeh, M.F. Hou, S.H. Wu, S.M. Tsai, S.K. Lin, L.A. Hou, H. Ma, L.Y. Tsai, Cell Biochem. Funct. 24 (2006) 555-559.

[83] I.S. Turan, F.P. Cakmak, D.C. Yildirim, R. Cetin-Atalay, E.U. Akkaya, Chemistry 20 (2014) 16088-16092.

[84] H. Shi, W. Sun, C. Liu, G. Gu, B. Ma, W. Si, N. Fu, Q. Zhang, W. Huang, X. Dong, J. Mater. Chem. B 4 (2016) 113-120.

[85] A.M. Durantini, L.E. Greene, R. Lincoln, S.R. Martinez, G. Cosa, J. Am. Chem. Soc. 138 (2016) 1215-1225.

[86] P.A. de Silva, N.H. Gunaratne, C.P. McCoy, Nature 364 (1993) 42-44.

[87] S. Ozlem, E.U. Akkaya, J. Am. Chem. Soc. 131 (2009) 48-49. 
[88] S. Erbas-Cakmak, F.P. Cakmak, S.D. Topel, T.B. Uyar, E.U. Akkaya, Chem. Commun. (Camb.) 51 (2015) 12258-12261.

[89] X.J. Jiang, J.T. Lau, Q. Wang, D.K. Ng, P.C. Lo, Chemistry 22 (2016) 8273-8281.

[90] D.K. Chatterjee, L.S. Fong, Y. Zhang, Adv. Drug Deliv. Rev. 60 (2008) 1627 1637.

[91] S.S. Lucky, K.C. Soo, Y. Zhang, Chem. Rev. 115 (2015) 1990-2042.

[92] J. Tian, L. Ding, H.J. Xu, Z. Shen, H. Ju, L. Jia, L. Bao, J.S. Yu, J. Am. Chem. Soc. 135 (2013) 18850-18858.

[93] A.S. Derycke, P.A. de Witte, Adv. Drug Deliv. Rev. 56 (2004) 17-30.

[94] E.C. Dreaden, M.A. Mackey, X. Huang, B. Kang, M.A. El-Sayed, Chem. Soc. Rev. 40 (2011) 3391-3404.

[95] M. González-Béjar, M. Liras, L. Francés-Soriano, V. Voliani, V. Herranz-Pérez, M. Duran-Moreno, J.M. Garcia-Verdugo, E.I. Alarcon, J.C. Scaiano, J. PérezPrieto, J. Mater. Chem. B 2 (2014) 4554-4563.

[96] B. Kilic, N. Yesilgul, V. Polat, Z. Gercek, E.U. Akkaya, Tetrahedron Lett. 57 (2016) 1317-1320.

[97] W.M. Sharman, J.E. van Lier, C.M. Allen, Adv. Drug Deliv. Rev. 56 (2004) 5376.

[98] H. Koo, M.S. Huh, I.C. Sun, S.H. Yuk, K. Choi, K. Kim, I.C. Kwon, Acc. Chem. Res. 44 (2011) 1018-1028.

[99] L. Liu, Z. Ruan, T. Li, P. Yuan, L. Yan, Biomater. Sci. 4 (2016) 1638-1645.

[100] Q. Zhang, Y. Cai, X.J. Wang, J.L. Xu, Z. Ye, S. Wang, P.H. Seeberger, J. Yin, A.C.S. Appl, Mater. Interfaces 8 (2016) 33405-33411.

[101] L. Huang, Z. Li, Y. Zhao, Y. Zhang, S. Wu, J. Zhao, G. Han, J. Am. Chem. Soc. 138 (2016) 14586-14591.

[102] C.A. Robertson, D.H. Evans, H. Abrahamse, J. Photochem. Photobiol., B 96 (2009) $1-8$

[103] M. Pazos, H.B. Nader, Braz. J. Med. Biol. Res. 40 (2007) 1025-1035.

[104] D. Nowis, T. Stoklosa, M. Legat, T. Issat, M. Jakobisiak, J. Golab, Photodiagnosis Photodyn. Ther. 2 (2005) 283-298.

[105] E. Buytaert, M. Dewaele, P. Agostinis, Biochim. Biophys. Acta 1776 (2007) 86107.

[106] T. Yano, S. Takahashi, T. Ichikawa, Res. Commun. Mol. Pathol. Pharmacol. 87 (1995) 367-370.

[107] W.G. Roberts, M.W. Berns, Lasers Surg. Med. 9 (1989) 90-101.

[108] H. He, P.C. Lo, S.L. Yeung, W.P. Fong, D.K. Ng, Chem. Commun. (Camb.) 47 (2011) 4748-4750.

[109] Y.C. Lai, S.Y. Su, C.C. Chang, A.C.S. Appl, Mater. Interfaces 5 (2013) 1293512943.

[110] P. Verwilst, C.C. David, V. Leen, J. Hofkens, P.A. de Witte, W.M. De Borggraeve, Bioorg. Med. Chem. Lett. 23 (2013) 3204-3207.

[111] K. Mitra, S. Gautam, P. Kondaiah, A.R. Chakravarty, ChemMedChem 11 (2016) 1956-1967.
[112] A. Bhattacharyya, A. Dixit, K. Mitra, S. Banerjee, A.A. Karande, A.R. Chakravarty, MedChemComm 6 (2015) 846-851.

[113] J.M. Zen, S.L. Liou, A.S. Kumar, M.S. Hsia, Angew. Chem. Int. Ed. Engl. 42 (2003) 577-579.

[114] K. Zeitler, Angew. Chem. Int. Ed. Engl. 48 (2009) 9785-9789.

[115] D. Ravelli, D. Dondi, M. Fagnoni, A. Albini, Chem. Soc. Rev. 38 (2009) 19992011.

[116] M.A. Ischay, M.E. Anzovino, J. Du, T.P. Yoon, J. Am. Chem. Soc. 130 (2008) $12886-12887$.

[117] J. Du, T.P. Yoon, J. Am. Chem. Soc. 131 (2009) 14604-14605.

[118] A.G. Condie, J.C. Gonzalez-Gomez, C.R. Stephenson, J. Am. Chem. Soc. 132 (2010) 1464-1465.

[119] E. Baciocchi, T. Del Giacco, F. Elisei, M.F. Gerini, M. Guerra, A. Lapi, P. Liberali, J. Am. Chem. Soc. 125 (2003) 16444-16454.

[120] E. Baciocchi, T. Boschi, C. Galli, A. Lapi, P. Tagliatesta, Tetrahedron 53 (1997) 4497-4502.

[121] S.M. Bonesi, M. Fagnoni, A. Albini, Eur. J. Org. Chem. 2008 (2008) 2612-2620.

[122] M. Neumann, S. Fuldner, B. Konig, K. Zeitler, Angew. Chem. Int. Ed. Engl. 50 (2011) 951-954.

[123] I. Fernandez, N. Khiar, Chem. Rev. 103 (2003) 3651-3705.

[124] S. Caron, R.W. Dugger, S.G. Ruggeri, J.A. Ragan, D.H. Ripin, Chem. Rev. 106 (2006) 2943-2989.

[125] K.C. Agarwal, Med. Res. Rev. 16 (1996) 111-124.

[126] N. Maezaki, A. Sakamoto, N. Nagahashi, M. Soejima, Y.X. Li, T. Imamura, N. Kojima, H. Ohishi, K. Sakaguchi, C. Iwata, T. Tanaka, J. Org. Chem. 65 (2000) 3284-3291.

[127] J. Liang, C. Gu, M. Kacher, C.S. Foote, J. Am. Chem. Soc. 105 (1983) 4717-4721.

[128] E.L. Clennan, Acc. Chem. Res. 34 (2001) 875-884.

[129] W. Li, Z. Xie, X. Jing, Catal. Commun. 16 (2011) 94-97.

[130] L. Quan, W. Lin, T. Sun, Z. Xie, Y. Huang, X. Jing, Catal. Lett. 144 (2014) 308313.

[131] L. Wang, J. Cao, J.-W. Wang, Q. Chen, A.-J. Cui, M.-Y. He, RSC Adv. 4 (2014) $14786-14790$.

[132] P. Yang, J. Zhao, W. Wu, X. Yu, Y. Liu, J. Org. Chem. 77 (2012) 6166-6178.

[133] L. Huang, J. Zhao, S. Guo, C. Zhang, J. Ma, J. Org. Chem. 78 (2013) 5627-5637.

[134] N. Adarsh, M. Shanmugasundaram, R.R. Avirah, D. Ramaiah, Chemistry 18 (2012) 12655-12662.

[135] Z. Zhou, W. Yang, Synth. Commun. 44 (2014) 3189-3198.

[136] Y. Zhou, Z. Zhou, Y. Li, W. Yang, Catal. Commun. 64 (2015) 96-100.

[137] L. Huang, X. Yu, W. Wu, J. Zhao, Org. Lett. 14 (2012) 2594-2597.

[138] L. Huang, X. Cui, B. Therrien, J. Zhao, Chemistry 19 (2013) 17472-17482. 